\title{
Decisiones financieras, competencia en el mercado y desempeño de las empresas: Evidencia empírica para Iberoamérica
}

\author{
MANUEL SÁNCHEZ-VALADEZ \\ Dirección de Análisis Macroeconómico, BANCO DE MÉXICO, MÉXICO. E-mail: \\ manuel.sanchez@banxico.org.mx
}

\begin{abstract}
RESUMEN
La literatura económica ha mostrado la existencia de la interrelación entre las decisiones financieras de las empresas y sus decisiones competitivas. Sin embargo, ya sea por conveniencia, o por poca disponibilidad de datos, en muchos trabajos empíricos se han analizado separadamente la influencia de ambos tipos de decisiones sobre el desempeño de las empresas. Con ello, en este documento, a través de un modelo de sección cruzada, se exploran conjuntamente los posibles efectos que tienen sobre el desempeño de las empresas la competencia en el mercado y sus decisiones financieras, para ello se utilizan datos de alrededor de 3,500 empresas en 14 países de Iberoamérica. Los resultados hallados apuntan a la existencia de diferencias en las relaciones entre las variables, de acuerdo a la intensidad de la competencia que enfrentan las empresas. Asimismo, los resultados sugieren que las decisiones financieras de las empresas podrían estar siendo utilizadas por éstas como un instrumento más de su estrategia competitiva.
\end{abstract}

Palabras clave: Endeudamiento, crédito comercial, competencia, desempeño.

\section{Financial Decisions, Market Competition and Firm Performance: Empirical Evidence for Ibero-American Countries}

\begin{abstract}
Economic literature had shown the existence of the interrelationship between the financial decisions of the firms and their competitive decisions; either by convenience or by data availability, most of empiric papers addressed separately the influence of both kinds of decisions over firm performance. With it, this paper through a cross-section model, which uses information of around 3,500 enterprises in 14 Iberoamerican countries, explores jointly the possible effects of both kinds of decisions of the firms (financial and competitive) over their performance. The results suggest the existence of differences in the relationships between variables accordingly the market competition intensity. Also the results suggest that the financial decisions of the firms could be used as an additional tool of the competitive strategy of the firms.
\end{abstract}

Keywords: Indebtedness, Trade Credit, Competition, Firm Performance.

Clasificación JEL: G32, L20

\footnotetext{
${ }^{*}$ Las opiniones y errores en este documento son responsabilidad del autor y no reflejan el punto de vista de la institución donde labora. Se agradecen los comentarios de Francesc Trillas, Miguel Á. García-Cestona, Xavier Fageda y de los dictaminadores anónimos a una versión previa del documento.
}

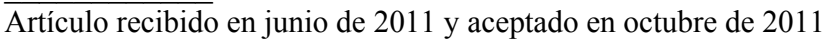

Artículo disponible en versión electrónica en la página www.revista-eea.net, ref. ə-30107 


\section{INTRODUCCIÓN}

Genéricamente, las decisiones de las empresas pueden clasificarse en decisiones de producción o decisiones financieras. Las primeras tienen que ver con la elección de precios y cantidades, y se identifican, clásicamente, con la estrategia competitiva de las empresas en los mercados. Las segundas reflejan la preferencia de los niveles de financiación y la elección de los instrumentos utilizados para ello. Éstas últimas también pueden formar parte de la estrategia competitiva de las empresas (Neff (2003)). Aybar-Arias et al. (2003), haciendo alusión a los enfoques que determinan la estructura de capital de las empresas, mencionan que la estrategia empresarial afecta de diversas maneras a la estructura del capital. Por lo que la forma cómo enfrentan la competencia, puede influir sobre el nivel del endeudamiento de las empresas o el mayor uso de ciertos instrumentos en la financiación de las mismas. Así, en la determinación del entorno competitivo donde interactúan las empresas pueden incidir tanto las decisiones sobre la producción y los precios, como sus decisiones financieras.

Respecto a los efectos generados por las diferentes decisiones de las empresas sobre su desempeño, en la literatura se ha propuesto la existencia de un efecto positivo entre la diversidad de fuentes de financiamiento que puede tener una empresa y su desempeño (Levine (2004), Dermiguc-Kunt y Maksimovic (2001) y Beck et al. (2008)), e incluso se ha mostrado que los efectos de las decisiones financieras sobre el desempeño dependerán del sector al que pertenece la propia empresa (Fisman y Love (2003)). ${ }^{1}$

Sin embargo, los efectos que la competencia en el mercado pueda tener sobre el desempeño de las empresas no son del todo claros. Aghion y Griffith (2005) apuntan la existencia de dos efectos contrapuestos, lo que resulta en una relación en forma de "U invertida" entre la competencia y la innovación, estableciendo esta última una relación directa con el crecimiento de las empresas. Uno de los efectos, el efecto Schumpeteriano, implica que un incremento de la competencia en el mercado disminuye los incentivos para innovar, y con ello las empresas reducen su crecimiento. El otro efecto, denominado efecto de escape de la competencia -escape competition effect-, señala que el incremento de la competencia en el mercado induce a las empresas en el mercado a innovar, propiciando con ello su crecimiento. El dominio de uno u otro efecto sobre el desempeño de las empresas dependerá de las características tecnológicas del sector o la industria a la que pertenecen.

No obstante la interrelación existente entre las decisiones financieras de las empresas y sus decisiones competitivas, ya sea por conveniencia o por disponibilidad de datos, diversos trabajos empíricos han abordado separadamente la

\footnotetext{
${ }^{1}$ Entendiendo como decisión financiera de la empresa, el uso de una determinada fuente de financiamiento.
} 
influencia que tienen ambos tipos de decisiones sobre el desempeño de las empresas. A diferencia de trabajos previos, en este documento se contrastaran diferentes hipótesis vinculadas entre sí por los efectos que tienen sobre el desempeño de las empresas, sus decisiones financieras -y con ello el acceso a fuentes de financiamiento- y la competencia en el mercado. Es claro que ambos elementos pueden condicionar la permanencia de las empresas en el mercado. En este sentido, una condición necesaria para que las empresas sigan produciendo, es contar con los recursos financieros suficientes, los cuales pueden condicionar la permanencia y desempeño en los mercados (Fisman (2001) y Saeed (2009)). Con ello, una primera hipótesis plantea que: El acceso a diversas fuentes de financiamiento y la competencia en el mercado tiene un efecto positivo sobre el desempeño de las empresas.

Otro elemento que condiciona la permanencia y desempeño de una empresa en el mercado, es la intensidad de la competencia a la que se enfrenta. Como se mencionó, en la literatura se ha planteado la existencia de dos efectos contrapuestos de la competencia sobre el desempeño de las empresas, guardando este último una relación directa con la innovación (Aghion y Griffith (2005)). Un primer efecto (efecto de escape de la competencia o efecto Darwiniano) plantea que ante un mayor grado de competencia en el mercado las empresas podrían obtener mayores beneficios, y por lo tanto mejorar su desempeño. Lo anterior como resultado de reducciones en los costos y la introducción de innovaciones en la producción. ${ }^{2}$ En el mismo sentido, Boone (2008) propone que ante una mayor competencia en el mercado se genera un mecanismo de redistribución de beneficios, en el cual los ingresos de las empresas menos eficientes se ven disminuidos a favor de las empresas más eficientes (profit reallocation effect of competition). De funcionar este último mecanismo, la competencia en el mercado tendría un efecto positivo sobre el desempeño de las empresas que sobreviven, al desplazar del mercado a las empresas menos eficientes. Un segundo efecto planteado (efecto Schumpeteriano), propone que la competencia genera incertidumbre en el mercado, traduciéndose en una menor innovación y con ello en un desempeño más pobre de las empresas. La predominación de un efecto u otro dependerá de las diferencias tecnológicas entre las empresas del sector o industria. ${ }^{3}$ Considerando el mecanismo de redistribución de beneficios propuesto por Boone (2008) y la existencia del efecto Darwiniano de la competencia, y asumiendo que en las economías en cuestión los sectores que tienen

\footnotetext{
${ }^{2}$ Salas (1996) menciona que una empresa podrá verse favorecida de su mayor competitividad, siempre que demuestre suficiente capacidad innovadora y asiente la innovación en recursos y habilidades difíciles de imitar o reproducir por otras empresas.

${ }^{3}$ De acuerdo con Aghion y Griffith (2005), en sectores con grandes disparidades tecnológicas dominará el efecto negativo. Mientras que para la economía en su conjunto, el efecto predominante dependerá de la distribución de las características tecnológicas entre los diferentes sectores de la economía.
} 
niveles tecnológicos similares son mayoría, la segunda hipótesis plantea que: La competencia en el mercado tiene un efecto positivo sobre el desempeño de las empresas.

Considerando lo anterior, y como se mencionó, ante la existencia de presiones competitivas en el mercado, al enfrentarlas con el fin de permanecer en el mercado y/o mejorar su desempeño, las empresas pueden hacer uso de diversos instrumentos a su alcance, y entre éstos los instrumentos financieros. En este sentido, las diferencias en las presiones competitivas en el mercado podrían incidir tanto en el desempeño de las empresas, como en sus decisiones financieras. ${ }^{4}$ Así, una tercera hipótesis plantea que: Frente a un entorno competitivo más agresivo, y con el fin de mejorar su desempeño para permanecer en el mercado, las empresas utilizarán los instrumentos financieros disponibles como un elemento más de sus estrategias competitivas.

Las hipótesis planteadas, se contrastan utilizando datos de empresas en Argentina, Bolivia, Chile, Colombia, Ecuador, El Salvador, Guatemala, Honduras, México, Nicaragua, Panamá, Paraguay, Perú y Uruguay, contenidos en la Encuesta de las Empresas -Enterprise Survey (ES)- llevada a cabo por el Banco Mundial en 2006. Genéricamente, los resultados sugieren que de acuerdo a la intensidad de la competencia enfrentada en el mercado difieren las relaciones de las variables que determinan el desempeño de las empresas; que el acceso al sistema financiero se relaciona positivamente con el desempeño de las empresas $\mathrm{y}$; que las decisiones financieras de las empresas podrían estar siendo utilizadas como un instrumento adicional dentro de su estrategia competitiva.

El resto del documento se compone de cuatro secciones más. La segunda sección es una revisión de algunos trabajos previos sobre el tema. En la tercera sección se exponen la metodología sugerida y los datos utilizados en las estimaciones. Mientras que en la cuarta sección se explican los resultados hallados y la quinta, y última sección, contiene las conclusiones.

\section{REVISIÓN BIBLIOGRÁFICA}

El desempeño de las empresas se ha abordado desde diferentes perspectivas. En algunos casos éste es visto como un concepto sinónimo al crecimiento de las empresas, mientras que en otros se asocia más a la productividad de las mismas. En todo caso, el desempeño de las empresas es visto como un cambio en los beneficios de las empresas, el cual puede ser una mejoría o un deterioro.

La disponibilidad de datos a nivel de empresa ha permitido que la literatura empírica al respecto haya crecido. Sin embargo, como Coad y Hölzl (2010)

\footnotetext{
${ }^{4}$ Existe evidencia empírica sobre un efecto diferenciado en el comportamiento financiero de las empresas ante diversos tipos e intensidad de las presiones competitivas en el mercado, entre otros Showalter (1999), Lyandres (2006), Jong et al. (2007) y Sánchez-Valadez (2009, 2010).
} 
mencionan, los resultados hallados muestran que el crecimiento de las empresas está altamente asociado con aspectos idiosincrásicos y es difícil de predecir. De esta manera, algunos documentos se han orientado a explorar los efectos de la competencia en el mercado sobre el desempeño de las empresas, mientras que otros han fijado su atención en los efectos que tienen el acceso y la utilización de diversas fuentes de financiamiento sobre el desenvolvimiento de éstas.

En la revisión de la literatura sobre el tema sin lugar a dudas un documento importante al respecto es el de Nickell (1996). Este autor sugiere la existencia de un efecto positivo de la competencia en el mercado sobre el desempeño de las empresas manufactureras en el Reino Unido. En el mismo sentido, Tang y Wang (2002) aportan evidencia de la existencia de un efecto positivo de la competencia en el mercado sobre la productividad de las empresas manufactureras en Canadá. Aghion et al. (2008) documentan la relación entre competencia y productividad para el caso de empresas en Sudáfrica, corroborando la existencia de un efecto positivo de la competencia sobre la productividad de las empresas. Por su parte, Salgado y Bernal (2007) sugieren la existencia de una relación negativa entre la concentración de mercado -menor competencia- y la productividad para el caso de las empresas manufactureras en México. Mientras que Maroto et al. (2006) analizan los efectos del grado de competencia en el mercado y la presión de la deuda sobre la productividad de una muestra de empresas españolas, hallando un efecto positivo de ambas variables.

Otro grupo de trabajos, aprovechan la información de algunas encuestas a empresas en economías en transición, elaboradas por el Banco Mundial, y analizan los citados efectos entre la competencia y el desempeño de las empresas. Entre otros, Carlin et al. (2004) quienes aportan evidencia sobre la influencia que tienen diferentes grados de presión competitiva sobre el desempeño de las empresas, medido éste último a través del crecimiento de las ventas. Carlin et al. (2001) además de analizar las relaciones entre la competencia en el mercado y el desempeño de las empresas, exploran también los efectos que tiene la estructura de propiedad de las empresas sobre su desempeño; sus resultados muestran que la competencia tiene un efecto no monotónico sobre el crecimiento de las ventas de las empresas y la productividad del trabajo. Commander y Svejnar (2008) hallan que la competencia en aquellas empresas propiedad de extranjeros afecta positivamente el desempeño de las empresas. Ospina y Schiffbauer (2010) encuentran que países que implementaron reformas estructurales en los mercados de productos, tienen más competencia en el mercado, lo cual genera mayores incrementos en la productividad de las empresas.

Un comentario aparte merece el documento de Aghion et al. (2005), quienes con datos de una muestra de empresas en el Reino Unido aportan evidencia sobre la existencia de una relación en forma de "U invertida" entre la competencia y la innovación, guardando esta última una relación directa con el creci- 
miento de las empresas. Aghion y Griffith (2005) explican que la forma de "U invertida" hallada por Aghion et al. (2005) es resultado a su vez de la presencia de dos efectos: el efecto de escape de la competencia -o efecto Darwiniano- y el efecto Schumpeteriano de la competencia. El primero representa el efecto positivo, y se origina cuando las empresas innovan con el fin de no enfrentar la competencia en el mercado, lo que facilita el crecimiento productivo de las empresas. Este efecto se origina porque la competencia en el mercado afecta los ingresos de las empresas dominantes antes de la innovación -pre-innovation rents- más no los ingresos obtenidos después de la innovación -post-innovation rents-, con lo cual la empresa tiene incentivos para innovar, lo que le permite eludir a la competencia y mejorar su desempeño. El segundo -el efecto Schumpeteriano- es el efecto negativo de la competencia, bajo el cual la mayor competencia en el mercado genera incertidumbre sobre las rentas futuras de las empresas, lo que desalienta la innovación e inhibe el crecimiento productivo de las empresas, a través de la reducción de sus ingresos.

Por otra parte, algunos documentos se han enfocado en revisar los efectos que tienen el acceso y la utilización de diferentes fuentes de financiamiento sobre el desempeño de las empresas. Al respecto, Nickell et al. (1997) y Maroto et al. (2006) analizan los efectos que tiene la presión financiera sobre la productividad de las empresas, los resultados de ambos trabajos aportan evidencia que apunta a la existencia de un efecto positivo de la presión financiera sobre el comportamiento de las empresas. Por su parte los resultados de Gohsh (2009) muestran que, en promedio, las empresas con un menor apalancamiento son más productivas, denotando de esta manera la relación entre la estructura financiera de las empresas y el desempeño de las mismas.

Otros documentos centran la atención en el papel que tiene la utilización de instrumentos financieros específicos, como el crédito comercial, sobre el desempeño de las empresas. Al respecto, los resultados de Fisman (2001) sugieren la existencia de un efecto positivo del acceso al crédito comercial sobre la eficiencia productiva de las empresas. Fisman y Love (2003) muestran que empresas en sectores con gran dependencia del crédito comercial tienen tasas de crecimiento mayores en países que carecen de instituciones financieras consolidadas. ${ }^{5} \mathrm{La}$ evidencia hallada por Li y Lu (2008) muestra que ante mercados financieros poco desarrollados el crédito comercial ayuda a las empresas a crecer más rápido. Y Cull et al. (2009) muestran que las empresas privadas productivas son más propensas a otorgar crédito en comparación con las empresas menos productivas.

Por otra parte, algunos documentos han explorado los efectos del acceso a

\footnotetext{
${ }^{5}$ El documento de Fisman y Love (2003) se relaciona también con la literatura que propone la existencia de un efecto positivo del desarrollo del sistema financiero sobre el crecimiento económico, al respecto, Levine (2004) constituye una referencia básica.
} 
fuentes externas de financiamiento sobre el desempeño de las empresas. Gatti y Love (2008) aportan evidencia sobre el papel del acceso al crédito en la productividad para el caso de las empresas en Bulgaria, demostrando que el acceso al crédito está asociado positivamente con la productividad total de los factores. Brown et al. (2008), con datos de empresas en cerca de 70 países en desarrollo, confirman que el acceso a fuentes externas de capital se relaciona con el crecimiento de las empresas. Butler y Cornaggia (2008) utilizando información de empresas en 12 estados del medio-oeste de los Estados Unidos, corroboran la evidencia empírica respecto al efecto positivo del financiamiento en el desempeño de las empresas. Por su parte Saeed (2009), considerando datos de una muestra de empresas en diversas economías en transición, analiza los efectos del uso de diferentes fuentes de financiamiento sobre el desempeño de las empresas, mostrando que el crédito comercial y el acceso los recursos de bancos locales mejoran el crecimiento de las empresas pequeñas y medianas.

Cabe mencionar que la mayoría de los documentos revisados, se centran en explorar los efectos de la competencia o las decisiones financiera de las empresas sobre su productividad, la cual como se ha mencionado es considerada un sinónimo del desempeño de las empresas. Sin embargo, el cálculo de una medida específica de la productividad de las empresas supera los alcances de este trabajo, por lo tanto el proxy de desempeño de las empresas a utilizarse en las diferentes estimaciones (ventas de las empresas) no pretende ser un sustituto de una medida de la productividad de las mismas.

De acuerdo con los diferentes documentos citados, es posible apreciar la existencia de un efecto positivo de la disponibilidad de diversas fuentes de financiamiento sobre el desempeño de las empresas. Es decir, que ante mayor variedad de fuentes alternativas de financiamiento las empresas verán favorecido su desempeño. Mientras que en el caso de los efectos de la competencia en el mercado, los documentos analizados aportan evidencia tanto de un efecto positivo de la competencia sobre el desempeño de las empresas, como de la coexistencia de efectos contrarios de la competencia sobre la actuación de las empresas -la forma de "U invertida" propuesta por Aghion et al. (2005). ${ }^{6}$

\section{METODOLOGÍA EMPÍRICA, DATOS Y VARIABLES}

Este documento propone tres hipótesis a contrastar, las cuales se interrelacionan por los efectos que tienen sus decisiones financieras y la competencia en el mercado sobre el desempeño de las empresas. Así, las dos primeras plantean contrastar los efectos del acceso a las fuentes de financiamiento y la compe-

\footnotetext{
${ }^{6}$ Cabe mencionar la vertiente de la literatura, de claro perfil macroeconómico, sobre la relación entre la competencia y el crecimiento económico. Aghion y Griffith (2005) mencionan algunos de estos modelos de crecimiento endógeno.
} 
tencia en el mercado sobre el desempeño de las empresas, respectivamente, y una tercera plantea la inclusión de las decisiones financieras como parte de la estrategia competitiva de las empresas.

Con lo anterior, tomando como referencia lo realizado por Commander y Svejnar (2008), en este documento se adopta una definición del desempeño de las empresas relacionada directamente con la obtención de ingresos. De esta manera, considerando una función de producción aumentada del tipo CobbDouglas, en este trabajo se estiman diversas especificaciones de un modelo econométrico de datos de sección cruzada, en las cuales se asume que el nivel de las ventas de las empresas, que es el proxy elegido sobre el desempeño de las empresas, está determinado por dos insumos principales, capital y trabajo, y por una serie de factores adicionales como la competencia en el mercado, el acceso a los recursos financieros y/o el entorno macroeconómico. ${ }^{7}$ Cabe señalar que la estructura de la información disponible condiciona la metodología utilizada, pues por país sólo se tiene una ronda de la encuesta utilizada y por lo tanto con esta información sólo es posible realizar estimaciones de sección cruzada.

Con lo anterior, dada la metodología a seguir (estimaciones de una función Cobb-Douglas ampliada) y el tipo de datos a utilizar (microdatos en sección cruzada), un problema metodológico a resolver es la potencial endogeneidad de una o más variables consideradas como independientes. Una estrategia usual en la literatura para resolverla, es la estimación de mínimos cuadrados utilizando variables instrumentales. Al respecto, Murray (2006) menciona que si bien la estimación a través de variables instrumentales parece la panacea al resolver una gran cantidad de problemas de los estimadores, el trabajo del investigador no es tan simple, puesto que "... all instruments arrive on the scene with a dark cloud of invalidity hanging overhead. This cloud never goes entirely away, but researchers should chase away as much of the cloud as they can." (Murray (2006, p.114)). Con ello, ante la potencial endogeneidad de las variables proxies de capital y trabajo éstas se instrumentaron, evitando así el sesgo y la inconsistencia de los estimadores. Asumiendo la exogeneidad de las demás variables de control incluidas en las estimaciones del modelo de sección cruzada, con el conjunto de datos disponibles se consideran diversas especificaciones de una función del tipo Cobb-Douglas aumentada como la siguiente:

$$
\begin{aligned}
& \log \left(C_{i}\right)=\alpha+\gamma_{i} Z_{i}+v_{i} \\
& \log \left(T_{i}\right)=\alpha+\gamma_{2} Z_{2}+v_{i} \\
& \log (\text { taş })=\alpha+\beta_{1} \log \left(C_{i}\right)+\beta_{2} \log \left(T_{i}\right)+\beta_{3} D F_{i}+\beta_{4} C O M P+\beta_{5} C E_{i}+\beta_{6} E M_{i}+\beta_{7} I_{i}+\varepsilon
\end{aligned}
$$

\footnotetext{
${ }^{7}$ Coad y Hölzl (2010) mencionan como determinantes del crecimiento de las empresas factores asociados a: la innovación, los beneficios, la productividad, las características propias de la empresa -como la educación de los empresarios o la estructura de propiedad de las empresas-, la industria perteneciente y el entorno macroeconómico.
} 
La tercera parte de la ecuación (1) define la especificación genérica del nivel de las ventas de las empresas (Vtas). Mientras que C y T son las variables de capital y trabajo estimadas en la primera y segunda parte de la ecuación, respectivamente. En éstas, $Z$ es un vector de variables que se asumen exógenas al nivel de ventas de la empresa. DF, es el vector de variables que capturan las decisiones financieras de las empresas. COMP, es el vector de variables que define los proxies sobre la competencia enfrentada en el mercado. CE, EM e I son vectores de variables que representan las características propias de la empresa (CE), el entorno macroeconómico enfrentado (EM) y la industria (I) a la que pertenece la empresa. No obstante, hay que reconocer que el concepto del desempeño de las empresas tiene un carácter plenamente dinámico. Por lo tanto, lo óptimo, siempre que se cuente con la información, sería la estimación de modelos dinámicos, lo que permitiría apreciar los cambios en los diferentes parámetros que definen el comportamiento de las empresas. Sin embargo, debido a la estructura de los datos disponibles, en este documento no es posible realizar estimaciones dinámicas. ${ }^{8}$

Los datos utilizados para contrastar las hipótesis propuestas, corresponden a la información de alrededor de 10.400 empresas en 14 países iberoamericanos, contenidos en encuestas llevadas a cabo en 2006 por el Banco Mundial - Enterprise Survey (ES)-. En dichas encuestas, las empresas reportan información tanto cuantitativa como cualitativa sobre las fuentes de financiamiento utilizadas, ventas, cantidad y características de la fuerza laboral, barreras de regulación en el mercado, grado de competencia, innovación, acceso a la infraestructura de servicios, productividad, clima de negocios en la economía y corrupción. ${ }^{9}$

En la construcción de la base de datos se aplicaron algunos filtros. Así, sólo se considera a las empresas manufactureras y; se excluyen a las empresas que no respondieron la pregunta sobre el valor de sus ventas, por lo que la muestra se integró por 5.894 empresas. Sin embargo la muestra final es menor, debido a la eliminación de empresas que no tienen respuestas en las preguntas utilizadas en la construcción de variables independientes. Con ello, la muestra final incluye sólo 3.512 empresas distribuidas de la siguiente manera: 635 mexicanas, 457 colombianas, 361 chilenas, 310 argentinas, 234 guatemaltecas, 222 ecuatoria-

\footnotetext{
${ }^{8}$ El análisis empírico realizado en este documento, como se describe al inicio de la siguiente sección, se basa en parte en el análisis y comparación del comportamiento de diferentes submuestras de empresas. Cabe aclarar, que con la información disponible, no es posible determinar causalidad entre las variables, por lo que en los resultados del documento, sólo se apunta la existencia de relaciones entre las mismas.

${ }^{9}$ En la construcción las muestras de estas encuestas -ES-, en algunos casos, se utiliza un muestreo aleatorio simple, y en otros un muestreo aleatorio estratificado (Banco Mundial (2007)). Información detallada sobre la metodología seguida en la construcción de las muestras de las ES puede encontrarse en la página web: http://www.enterprisesurveys.org/.
} 
nas, 209 salvadoreñas, 208 nicaragüenses, 191 peruanas, 183 bolivianas, 168 hondureñas, 125 paraguayas, 118 uruguayas y 91 panameñas.

La variable dependiente se definió como el logaritmo de las ventas de las empresas, expresadas en dólares de Estados Unidos (vtas), la cual intenta capturar los ingresos obtenidos por la empresa, por lo que de manera directa estaría expresando el nivel de beneficios obtenidos por la misma. Cabe comentar que en algunos de los documentos consultados, el comportamiento de las empresas se aproxima a través de sus ventas; por ejemplo, Nickell (1996), Carlin et al. $(2001,2004)$ y Commander y Svejnar (2008).

Los datos de la ES permiten construir dos diferentes conjuntos de proxies sobre el nivel de competencia en el mercado. Siguiendo lo sugerido por Carlin et al. (2001, 2004) y Sánchez-Valadez (2010), un primer conjunto de proxies se utilizaron las respuestas a la pregunta: ¿Cuántos competidores enfrenta en el principal mercado de su principal producto?, a partir de las respuestas se construyeron cuatro variables dicotómicas que reflejan diferentes grados de competencia. Así, la variable compl es igual a 1 si la empresa respondió no tener competidores en el mercado, comp 2 es igual a 1 si la empresa respondió tener un competidor en el mercado, comp 3 es igual a 1 si la empresa enfrenta entre 2 y 5 competidores y comp 4 es igual a 1 si la empresa se enfrenta a más de 5 rivales en el mercado. Por su parte, la variable comp agrupa las respuestas de las anteriores cuatro variables. Estas cinco variables intentan capturar las presiones competitivas que enfrentan las empresas al existir un mayor número de rivales en el mercado. Como se plantea en la segunda hipótesis propuesta, se espera que la mayor competencia tenga un efecto positivo sobre el desempeño de las empresas. De esta manera, ante más competencia, las empresas podrían implementar nuevas estrategias con el fin de permanecer en el mercado, tratando de obtener el máximo beneficio posible. ${ }^{10}$

Un segundo conjunto de proxies de la competencia en el mercado, se construyó con las respuestas de las empresas tanto sobre la mejora de los productos o procesos existentes o la introducción de nuevos productos. De nuevo, este conjunto de variables se construyó a partir de lo sugerido por Carlin et al. (2001, 2004), y lo componen dos variables dummy, la primera, nprod, es igual a 1 si la empresa respondió que hizo mejoras a los productos o introdujo nuevos productos en el mercado y cero en otro caso, la segunda variable, nproc, es igual a 1 si la empresa mejoró los procesos y cero en caso contrario. Este segúndo conjunto de variables intenta capturar las presiones competitivas que pueden desencadenar el proceso de innovación por parte de las empresas, y de esta manera reflejar la existencia del efecto Darwiniano de la competencia. Como se

${ }^{10}$ Esta variable está sujeta a crítica y mejora, pues como Boone (2008) menciona, la menor concentración, y por lo tanto un mayor número de empresas en el mercado no siempre captura la existencia de presiones competitivas en el mercado. 
planteó al inicio del documento, el efecto Darwiniano de la competencia propone que ésta tiene un efecto positivo sobre el desempeño de las empresas. Así, el incremento en la presión de los competidores podría traducirse en la introducción de mejoras, que podría a su vez generar un volumen mayor de ventas, y por lo tanto mayores beneficios; de esta manera la competencia en el mercado, capturada a través de la presión para innovar, se ve reflejada en una mejora del desempeño de las empresas. Es de comentar que estos últimos proxies sobre la competencia en el mercado (nprod y nproc), no sólo reflejan la existencia de competencia en el mercado, éstos incorporan también información sobre la intensidad de la competencia que enfrentan las empresas. Sin embargo, dado que estas variables se construyen a partir de las respuestas de las empresas, cabe la posibilidad de la existencia de un sesgo subjetivo.

Una manera de reflejar las decisiones financieras de las empresas es a través de la elección de un determinado instrumento en su financiación. Con ello, considerando lo sugerido por Sánchez-Valadez (2010) se construyeron cuatro variables dicotómicas tomando las respuestas sobre la estructura porcentual en la financiación del capital de trabajo de las empresas, el cual incluye los gastos de corto plazo que le permiten a la empresa continuar operando. Cada una de estas variables refleja la elección de un instrumento utilizado en la financiación. De manera general, se espera que todas tengan un efecto positivo sobre el desempeño de las empresas, puesto que a mayor diversidad de fuentes de financiamiento, es menor la probabilidad de interrupción de la cadena productiva, y por lo tanto se incrementa la posibilidad de obtener beneficios. Así, ct propios es igual a 1 si la empresa utilizó recursos propios para financiarse; ct_bancos igual a 1 refleja si la empresa utilizó recursos de algún banco comercial, o del estado, en la financiación del capital de trabajo.; ct_crcom, es igual a 1 si la empresa recurrió al crédito de sus proveedores para financiar su capital de trabajo; y por último, ct_otras recoge el uso de fuentes informales alternativas del financiamiento (amigos, familiares y otros prestamistas), por lo que ésta es igual 1 si la empresa utilizó estas fuentes. Cabe señalar que aún y cuando el crédito comercial podría clasificarse como una fuente informal de recursos, se decidió denotar explícitamente la utilización de esta fuente de recursos debido a la intensidad y frecuencia de su uso por parte de las empresas. ${ }^{11}$

El acceso a fuentes formales de recursos, es decir al sistema financiero formal, se capturó a través de una variable dummy (acces), construida con las respuestas a la pregunta: ¿Tiene usted posibilidad de sobregiro? Si la empresa contestó que sí, acces es igual 1 y cero en caso contrario. El acceso al sistema financiero formal, como lo menciona Levine (2004), se espera tenga un efecto

\footnotetext{
${ }^{11}$ Existe literatura vasta sobre la importancia del crédito comercial como fuente de financiamiento de las empresas, entre otros Demirguç-Kunt y Maksimovic (2001) analizan el uso del crédito comercial como fuente alternativa de recursos de las empresas.
} 
positivo sobre el desempeño de las empresas, puesto que la disponibilidad de recursos financieros permite a las empresas continuar produciendo.

Dado que se pretende estimar el nivel de ingresos de las empresas, como se mencionó, se asumió una forma simple de función de producción con dos insumos principales, capital y trabajo. El capital (cap), se aproximó con el logaritmo del valor de los activos fijos, denotados en dólares de los Estados Unidos de América. El trabajo (trab) se capturó con el logaritmo del número total de trabajadores de tiempo completo. ${ }^{12}$ Ante la posible endogeneidad de estas variables, siguiendo lo sugerido por Commander y Svejnar (2008), éstas se instrumentaron utilizando un conjunto de variables dummies que capturan la antigüedad de la empresa: edadl igual a 1 si la empresa tiene menos de seis años en el mercado, edad 2 igual a 1 si la empresa ha estado en el mercado entre 6 y 15 años y edad 3 igual a 1 si la empresa ha permanecido en el mercado por más de 15 años. Se incluyen también como instrumentos, una variable dicotómica igual a 1 si la empresa se localiza en la ciudad capital del país (dciud), una variable con el porcentaje de trabajadores cualificados en la empresa (edul), una variable con el logaritmo del número de trabajadores permanentes que la empresa declaró tener 3 años antes al levantamiento de la encuesta (trabt3) y la interacción de la variable edul tanto con las variables sobre la antigüedad de la empresa, como con la variable de localización de la misma. ${ }^{13}$

Por otra parte, la información en la ES permite controlar por otras características de las empresas y por el entorno de negocios donde interactúan. ${ }^{14}$ Dentro de las características de las empresas, se incluye una variable que refleja los años de experiencia en el sector del administrador de la empresa ( $a d m n)$, la cual se esperaría tuviese un efecto positivo sobre el desempeño de la misma, puesto que a mayor experiencia del administrador, mayor la probabilidad de un manejo eficiente y por lo tanto mayor probabilidad de mejorar los beneficios. Se incluye también una variable que captura si la empresa es exportadora (exp), ésta es una

${ }^{12}$ La ES define a los trabajadores de tiempo completo como aquellas personas contratadas por el término de un año fiscal, o más, que tienen garantizada la recontratación y que trabajan 8 o más horas al día.

${ }^{13}$ Los diferentes instrumentos considerados son buenos predictores de las dos variables potencialmente endógenas. Para las diferentes especificaciones del documento se realizaron pruebas estadísticas de endogeneidad de los regresores (Wu-Hausman y Durbin-Wu-Hausman), los resultados de estas pruebas para algunas especificaciones denotan que estadísticamente las variables cap y trab no son endógenas. Sin embargo, estos resultados estadísticos no son del todo coherentes con la intuición económica. Adicionalmente, y no obstante los resultados de las pruebas anteriores, se realizaron pruebas de sobreidentificación para verificar la validez de los instrumentos (Hansen).

${ }^{14}$ Carlin et al. (2001), Commander y Svejnar (2008) y Ospina y Schiffbauer (2010) para capturar el entorno de negocios, incluyen en sus estimaciones controles similares a los utilizados en este documento. 
variable dicotómica igual a 1 si la empresa reportó tener exportaciones directas o indirectas. Se espera un efecto positivo de esta variable, puesto que aquellas empresas que son exportadoras podrían mejorar su desempeño al tener más oportunidades de negocios por interactuar en mercados con diferente localización.

Como características del entorno de negocios, en las estimaciones se incluye una variable que refleja el tiempo requerido, medido en días, para iniciar un negocio en el país (apert). Esta variable se espera tenga un efecto negativo, puesto que mientras más días se requieran para iniciar un negocio, ello podría incidir en la expansión de las empresas y, por lo tanto influir negativamente en el desempeño de las mismas. Una variable más relacionada con el entorno de negocios es la variable ener, la cual es una variable dummy igual a 1 si la empresa sufrió algún corte en el suministro de agua o electricidad en el año, y cero en otro caso. Se esperaría que esta variable afecte negativamente el desempeño de la empresa, dado que la interrupción de estos insumos cortaría el proceso de producción de las empresas y con ello podrían disminuirse sus beneficios.

Por último, como lo sugieren Coad y Hölzl (2010), para controlar por rasgos estructurales de cada economía, en las estimaciones se incluyen la tasa de crecimiento porcentual del PIB en 2006, la cual pretende capturar la inercia del crecimiento que pueden presentar las actividades económicas no incluidas en la muestra; y la inflación anual en 2006, construida con los índices de precios al consumidor, base $2005=100$, reportados en las estadísticas financieras internacionales del Fondo Monetario Internacional. Esta última variable intenta capturar los posibles efectos del incremento de precios sobre el volumen de ventas de las empresas. Adicionalmente, con el fin de controlar por las posibles características específicas de cada industria -como ratios de utilización de capital o de trabajo-, se consideran dummies por cada una de las industrias incluidas en la muestra.

\section{RESULTADOS}

Para contrastar las hipótesis planteadas se realizaron diferentes ejercicios econométricos utilizando en todos ellos modelos de sección cruzada, sobre los cuales, en algunos casos, se realiza inferencia de acuerdo a las diferencias entre muestras. El primer ejercicio, consistió en la estimación del nivel de ventas de las empresas considerando una función de producción ampliada muy simple, donde como se mencionó, además de controlar por los insumos principales -capital y trabajo-, se incluyen controles sobre las características propias de las empresas, el entorno de negocios, el entorno macroeconómico, las fuentes de financiamiento disponibles y la competencia en el mercado. Una variante de este primer ejercicio, consistió en controlar las estimaciones de los ingresos de 
las empresas por el grado de desarrollo de las economías donde interactúan, para ello se realizaron algunas regresiones para tres subconjuntos de países, definidos éstos por el nivel del ingreso per-cápita. Un segundo conjunto de ejercicios econométricos consistió en la estimación de la misma función de producción ampliada para diversas sub-muestras. Las sub-muestras consideradas en este segundo conjunto de ejercicios, se construyeron a partir de las respuestas de las empresas sobre el número de competidores y la percepción acerca de las presiones competitivas en el mercado. En este sentido, estas últimas estimaciones intentan controlar los posibles efectos de la percepción sobre la intensidad de la competencia en el mercado, y con ello identificar, y analizar, la existencia de posibles relaciones existentes entre el desempeño de las empresas, la competencia en el mercado y el mayor, o menor, uso de los instrumentos financieros por parte de las empresas.

\subsection{Estimación de niveles de ventas}

El Cuadro 1 resume los resultados del primer ejercicio econométrico que considera los proxies de competencia construidos con las respuestas sobre el número de competidores en el mercado. En las diferentes especificaciones es posible apreciar la relativa importancia de la mano de obra como el insumo más importante en la determinación del nivel de ventas de las empresas. Por otra parte, los resultados sugieren la existencia de una relación positiva entre el acceso al sistema financiero y el desempeño de las empresas, lo cual es consistente con lo hallado por Gatti y Love (2008). Concretamente, el coeficiente del proxy sobre el acceso al sistema financiero es positivo en las diferentes especificaciones y, en todas, es estadísticamente significativo a un nivel elevado. Con ello, de estos resultados se podría inferir que, ante la posibilidad de contar con recursos del sistema financiero, las empresas podrían mejorar su desempeño a través del mejoramiento de sus condiciones de producción.

Respecto a las decisiones financieras de las empresas, solamente la utilización de recursos provenientes de otras fuentes informales guarda una relación negativa con el desempeño de las empresas, las demás fuentes de recursos muestran la existencia de una relación positiva, aunque sólo el crédito comercial es significativo estadísticamente en las diversas especificaciones. Concretamente, los resultados muestran que el efecto del crédito comercial sobre un cambio en el nivel de ventas supera en cerca de cuatro veces al efecto del uso de recursos propios, y en tres veces al uso de recursos provenientes de los bancos. Con ello, los resultados muestran la relativa importancia del crédito inter-empresarial para el funcionamiento de las empresas. En este sentido, estos resultados coinciden relativamente con lo hallado por Fisman (2001). 


\section{Cuadro 1}

Desempeño, decisiones financieras y número de competidores

\begin{tabular}{|c|c|c|c|c|c|c|c|c|c|c|}
\hline & \multicolumn{2}{|l|}{ (1) } & \multicolumn{2}{|c|}{ (2) } & \multicolumn{2}{|l|}{ (3) } & \multicolumn{2}{|l|}{ (4) } & \multicolumn{2}{|l|}{ (5) } \\
\hline Activos fijos & $\begin{array}{r}0,220 \\
(0,227) \\
\end{array}$ & & $\begin{array}{r}0,227 \\
(0,237) \\
\end{array}$ & & $\begin{array}{r}0,224 \\
(0,232) \\
\end{array}$ & & $\begin{array}{r}0,232 \\
(0,228) \\
\end{array}$ & & $\begin{array}{r}0,225 \\
(0,225) \\
\end{array}$ & \\
\hline $\begin{array}{l}\text { Trabajadores } \\
\text { permanentes }\end{array}$ & $\begin{array}{r}0,900 \\
(0,295)\end{array}$ & $\ddagger$ & $\begin{array}{r}0,886 \\
(0,306)\end{array}$ & $\ddagger$ & $\begin{array}{r}0,891 \\
(0,297)\end{array}$ & $\ddagger$ & $\begin{array}{r}0,885 \\
(0,295)\end{array}$ & $\ddagger$ & $\begin{array}{r}0,896 \\
(0,292)\end{array}$ & $\ddagger$ \\
\hline Actividad exportadora & $\begin{array}{r}0,251 \\
(0,203) \\
\end{array}$ & & $\begin{array}{r}0,263 \\
(0,199) \\
\end{array}$ & & $\begin{array}{r}0,263 \\
(0,199) \\
\end{array}$ & & $\begin{array}{r}0,244 \\
(0,200) \\
\end{array}$ & & $\begin{array}{r}0,241 \\
(0,202) \\
\end{array}$ & \\
\hline $\begin{array}{l}\text { Experiencia } \\
\text { administrador }\end{array}$ & $\begin{array}{r}-0,008 \\
(0,002) \\
\end{array}$ & $\dagger$ & $\begin{array}{r}-0,008 \\
(0,002) \\
\end{array}$ & $\dagger$ & $\begin{array}{r}-0,008 \\
(0,002) \\
\end{array}$ & $\dagger$ & $\begin{array}{r}-0,008 \\
(0,002) \\
\end{array}$ & $\dagger$ & $\begin{array}{r}-0,008 \\
(0,002) \\
\end{array}$ & $\dagger$ \\
\hline $\begin{array}{l}\text { Acceso al sistema } \\
\text { financiero }\end{array}$ & $\begin{array}{r}0,451 \\
(0,084) \\
\end{array}$ & $\dagger$ & $\begin{array}{r}0,459 \\
(0,081) \\
\end{array}$ & $\dagger$ & $\begin{array}{r}0,459 \\
(0,082) \\
\end{array}$ & $\dagger$ & $\begin{array}{r}0,448 \\
(0,082) \\
\end{array}$ & $\dagger$ & $\begin{array}{r}0,446 \\
(0,084) \\
\end{array}$ & $\dagger$ \\
\hline $\begin{array}{l}\text { Facilidades de } \\
\text { apertura }\end{array}$ & $\begin{array}{r}-0,004 \\
(0,004) \\
\end{array}$ & & $\begin{array}{r}-0,005 \\
(0,004) \\
\end{array}$ & & $\begin{array}{r}-0,005 \\
(0,004) \\
\end{array}$ & & $\begin{array}{r}-0,004 \\
(0,004) \\
\end{array}$ & & $\begin{array}{r}-0,004 \\
(0,004) \\
\end{array}$ & \\
\hline Cortes de energía & $\begin{array}{r}0,035 \\
(0,088) \\
\end{array}$ & & $\begin{array}{r}0,030 \\
(0,088) \\
\end{array}$ & & $\begin{array}{r}0,029 \\
(0,088) \\
\end{array}$ & & $\begin{array}{r}0,035 \\
(0,083) \\
\end{array}$ & & $\begin{array}{r}0,037 \\
(0,085) \\
\end{array}$ & \\
\hline $\begin{array}{l}\text { Uso de recursos } \\
\text { propios }\end{array}$ & $\begin{array}{r}0,063 \\
(0,039) \\
\end{array}$ & & $\begin{array}{r}0,069 \\
(0,039) \\
\end{array}$ & & $\begin{array}{r}0,069 \\
(0,038) \\
\end{array}$ & & $\begin{array}{r}0,060 \\
(0,043) \\
\end{array}$ & & $\begin{array}{r}0,059 \\
(0,042) \\
\end{array}$ & \\
\hline $\begin{array}{l}\text { Uso de recursos } \\
\text { bancarios }\end{array}$ & $\begin{array}{r}0,090 \\
(0,086)\end{array}$ & & $\begin{array}{r}0,089 \\
(0,084)\end{array}$ & & $\begin{array}{r}0,092 \\
(0,082)\end{array}$ & & $\begin{array}{r}0,081 \\
(0,086)\end{array}$ & & $\begin{array}{r}0,084 \\
(0,087)\end{array}$ & \\
\hline $\begin{array}{l}\text { Uso de crédito } \\
\text { comercial }\end{array}$ & $\begin{array}{r}0,277 \\
(0,083) \\
\end{array}$ & $\dagger$ & $\begin{array}{r}0,278 \\
(0,082) \\
\end{array}$ & $\dagger$ & $\begin{array}{r}0,282 \\
(0,082) \\
\end{array}$ & $\dagger$ & $\begin{array}{r}0,268 \\
(0,080) \\
\end{array}$ & $\dagger$ & $\begin{array}{r}0,271 \\
(0,081) \\
\end{array}$ & $\dagger$ \\
\hline Uso de otros recursos & $\begin{array}{r}-0,088 \\
(0,101) \\
\end{array}$ & & $\begin{array}{r}-0,095 \\
(0,108) \\
\end{array}$ & & $\begin{array}{r}-0,099 \\
(0,102) \\
\end{array}$ & & $\begin{array}{r}-0,086 \\
(0,103) \\
\end{array}$ & & $\begin{array}{r}-0,085 \\
(0,100) \\
\end{array}$ & \\
\hline Competencia (total) & $\begin{array}{r}-0,057 \\
(0,013) \\
\end{array}$ & $\dagger$ & - & & - & & - & & - & \\
\hline Sin competencia & - & & $\begin{array}{r}0,020 \\
(0,130) \\
\end{array}$ & & - & & - & & - & \\
\hline 1 competidor & - & & - & & $\begin{array}{r}0,258 \\
(0,150) \\
\end{array}$ & & - & & - & \\
\hline $\begin{array}{l}\text { Entre } 2 \text { y } 5 \\
\text { competidores }\end{array}$ & - & & - & & - & & $\begin{array}{r}0,153 \\
(0,041) \\
\end{array}$ & $\dagger$ & - & \\
\hline $\begin{array}{l}\text { Más de } 5 \\
\text { competidores }\end{array}$ & - & & - & & - & & - & & $\begin{array}{c}-0,184 \\
(0,034)\end{array}$ & $\dagger$ \\
\hline Constante & $\begin{array}{r}-0,010 \\
(0,543) \\
\end{array}$ & & $\begin{array}{r}2,101 \\
(0,597) \\
\end{array}$ & $\dagger$ & $\begin{array}{r}2,080 \\
(0,604) \\
\end{array}$ & $\dagger$ & $\begin{array}{r}1,938 \\
(0,581) \\
\end{array}$ & $\dagger$ & $\begin{array}{r}-0,139 \\
(0,575) \\
\end{array}$ & \\
\hline Observaciones & 3.520 & & 3.520 & & 3.520 & & 3.520 & & 3.520 & \\
\hline R2 (ajustada) & 0,745 & & 0,743 & & 0,744 & & 0,745 & & 0,745 & \\
\hline
\end{tabular}

Notas: Estimaciones utilizando el método de mínimos cuadrados en dos etapas. Las variables instrumentadas fueron las referentes a los activos fijos (cap) y los trabajadores permanentes (tra). En todas las especificaciones se incluyen como variables independientes el crecimiento del PIB y la inflación, se incluyen también dummies por industria. Se reportan entre paréntesis los errores estándar, estimados agrupando cluster- a las empresas por la industria a la que pertenecen. Coeficientes estadísticamente significativos al: $1 \%+, 5 \% \neq$ y $10 \%$ *.

Fuente: Estimaciones propias del autor con base en datos de la Enterprise Survey del Banco Mundial, Estadísticas del Banco Mundial y Estadísticas Monetarias y Financieras del Fondo Monetario Internacional.

Respecto a los efectos de la competencia en el mercado, los resultados del Cuadro 1 muestran relaciones diferentes de acuerdo al número de competidores enfrentados. Así, es posible ver que el coeficiente de la variable que captura la competencia de entre 2 y 5 competidores es positivo y significativamente esta- 
dístico, lo que contrasta con el coeficiente negativo y estadísticamente significativo de la variable que captura la mayor competencia posible en el mercado más de 5 competidores. Con ello, los resultados podrían estar indicando que las empresas se ven más favorecidas si enfrentan una competencia "moderada" en el mercado. Mientras que los coeficientes de las variables que capturan la competencia mínima o nula -un solo competidor o sin competidores-, si bien son positivos, éstos no son estadísticamente significativos. La diferencia en los coeficientes de los proxies de la competencia en las diversas especificaciones, sugieren que la competencia y el desempeño de las empresas guardan una relación en forma de "U invertida". En donde ante niveles bajos de competencia se muestra un ligero efecto positivo, el cual se incrementa y se mantiene positivo mientras que en el mercado halla menos de seis competidores, y se vuelve negativo ante mayores presiones competitivas. A partir de estos resultados, se podría proponer que la competencia "moderada" en el mercado mantiene una relación positiva con el desempeño de las empresas.

En el Cuadro 2 se resumen los resultados de las especificaciones que consideran como proxies de la competencia las variables asociadas con la introducción o la mejora de productos y/o procesos, las cuales intentan capturar las presiones competitivas originadas por los procesos de innovación. Estos resultados muestran que ambos insumos principales, capital y trabajo, obtienen el signo esperado y en la mayoría de las especificaciones son estadísticamente significativos. En este cuadro, de nueva cuenta, los resultados muestran que el acceso al sistema financiero guarda una relación positiva con el desempeño de las empresas, coincidiendo así con los resultados de la literatura previa que propone la existencia de un efecto positivo del acceso al sistema financiero sobre el crecimiento de las empresas, y por lo tanto de la economía.

\section{Cuadro 2}

Desempeño, decisiones financieras e innovación y competencia

\begin{tabular}{|c|c|c|c|c|c|c|c|c|}
\hline & \multicolumn{2}{|l|}{ (1) } & \multicolumn{2}{|l|}{ (2) } & \multicolumn{2}{|l|}{ (3) } & \multicolumn{2}{|l|}{ (4) } \\
\hline Activos fijos & $\begin{array}{r}0,318 \\
(0,160)\end{array}$ & * & $\begin{array}{r}0,319 \\
(0,162)\end{array}$ & * & $\begin{array}{r}0,215 \\
(0,216)\end{array}$ & & $\begin{array}{r}0,328 \\
(0,164)\end{array}$ & * \\
\hline $\begin{array}{l}\text { Trabajadores } \\
\text { permanentes }\end{array}$ & $\begin{array}{r}0,741 \\
(0,236)\end{array}$ & $\ddagger$ & $\begin{array}{r}0,741 \\
(0,233)\end{array}$ & $\dagger$ & $\begin{array}{r}0,914 \\
(0,296)\end{array}$ & $\ddagger$ & $\begin{array}{r}0,725 \\
(0,227)\end{array}$ & $\dagger$ \\
\hline Actividad exportadora & $\begin{array}{r}0,305 \\
(0,185) \\
\end{array}$ & & $\begin{array}{r}0,307 \\
(0,186) \\
\end{array}$ & & $\begin{array}{r}0,268 \\
(0,195) \\
\end{array}$ & & $\begin{array}{r}0,299 \\
(0,188) \\
\end{array}$ & \\
\hline Experiencia administrador & $\begin{array}{r}-0,009 \\
(0,002)\end{array}$ & $\dagger$ & $\begin{array}{r}-0,008 \\
(0,002)\end{array}$ & $\dagger$ & $\begin{array}{r}-0,008 \\
(0,002)\end{array}$ & $\dagger$ & $\begin{array}{r}-0,009 \\
(0,002)\end{array}$ & $\dagger$ \\
\hline $\begin{array}{l}\text { Acceso al sistema } \\
\text { financiero }\end{array}$ & $\begin{array}{r}0,483 \\
(0,099)\end{array}$ & $\dagger$ & $\begin{array}{r}0,488 \\
(0,098)\end{array}$ & $\dagger$ & $\begin{array}{r}0,457 \\
(0,079)\end{array}$ & $\dagger$ & $\begin{array}{r}0,483 \\
(0,100)\end{array}$ & $\dagger$ \\
\hline Facilidades de apertura & $\begin{array}{r}-0,004 \\
(0,003)\end{array}$ & & $\begin{array}{r}-0,004 \\
(0,003)\end{array}$ & & $\begin{array}{r}-0,003 \\
(0,003)\end{array}$ & & $\begin{array}{r}-0,005 \\
(0,003)\end{array}$ & \\
\hline Cortes de energía & $\begin{array}{r}0,024 \\
(0,069) \\
\end{array}$ & & $\begin{array}{r}0,020 \\
(0,067) \\
\end{array}$ & & $\begin{array}{r}0,032 \\
(0,087) \\
\end{array}$ & & $\begin{array}{r}0,025 \\
(0,069) \\
\end{array}$ & \\
\hline Uso de recursos propios & $\begin{array}{r}0,020 \\
(0,039)\end{array}$ & & $\begin{array}{r}0,019 \\
(0,039)\end{array}$ & & $\begin{array}{r}0,059 \\
(0,038)\end{array}$ & & $\begin{array}{r}0,022 \\
(0,043)\end{array}$ & \\
\hline
\end{tabular}


Cuadro 2 (continuación)

Desempeño, decisiones financieras e innovación y competencia

\begin{tabular}{|c|c|c|c|c|c|c|c|c|}
\hline & \multicolumn{2}{|l|}{ (1) } & \multicolumn{2}{|l|}{ (2) } & \multicolumn{2}{|l|}{ (3) } & \multicolumn{2}{|l|}{ (4) } \\
\hline Uso de recursos bancarios & $\begin{array}{r}0,074 \\
(0,077) \\
\end{array}$ & & $\begin{array}{r}0,082 \\
(0,080) \\
\end{array}$ & & $\begin{array}{r}0,106 \\
(0,097) \\
\end{array}$ & & $\begin{array}{r}0,069 \\
(0,069) \\
\end{array}$ & \\
\hline Uso de crédito comercial & $\begin{array}{r}0,306 \\
(0,065) \\
\end{array}$ & $\dagger$ & $\begin{array}{r}0,306 \\
(0,071) \\
\end{array}$ & $\dagger$ & $\begin{array}{r}0,282 \\
(0,077) \\
\end{array}$ & $\dagger$ & $\begin{array}{r}0,306 \\
(0,070) \\
\end{array}$ & $\dagger$ \\
\hline Uso de otros recursos & $\begin{array}{r}-0,099 \\
(0,079) \\
\end{array}$ & & $\begin{array}{r}-0,104 \\
(0,080) \\
\end{array}$ & & $\begin{array}{r}-0,095 \\
(0,101) \\
\end{array}$ & & $\begin{array}{r}-0,096 \\
(0,079) \\
\end{array}$ & \\
\hline Competencia (total) & - & & - & & $\begin{array}{r}-0,057 \\
(0,013) \\
\end{array}$ & $\dagger$ & - & \\
\hline $\begin{array}{l}\text { Introducción o mejora de } \\
\text { producto }\end{array}$ & $\begin{array}{r}-0,065 \\
(0,159) \\
\end{array}$ & & - & & $\begin{array}{r}-0,075 \\
(0,183) \\
\end{array}$ & & - & \\
\hline Mejora de procesos & - & & $\begin{array}{r}-0,102 \\
(0,088) \\
\end{array}$ & & $\begin{array}{r}-0,084 \\
(0,046) \\
\end{array}$ & * & - & \\
\hline Constante & $\begin{array}{r}2,174 \\
(0,521) \\
\end{array}$ & $\dagger$ & $\begin{array}{r}2,170 \\
(0,527) \\
\end{array}$ & $\dagger$ & $\begin{array}{r}2,231 \\
(0,595) \\
\end{array}$ & $\dagger$ & $\begin{array}{r}2,166 \\
(0,518) \\
\end{array}$ & $\dagger$ \\
\hline Observaciones & 3.926 & & 3.926 & & 3.512 & & 3.930 & \\
\hline R2 (ajustada) & 0,747 & & 0,746 & & 0,744 & & 0,746 & \\
\hline
\end{tabular}

Notas: Estimaciones utilizando el método de mínimos cuadrados en dos etapas. Las variables instrumentadas fueron las referentes a los activos fijos (cap) y los trabajadores permanentes (tra). En todas las especificaciones se incluyen como variables independientes el crecimiento del PIB y la inflación, se incluyen también dummies por industria. Se reportan entre paréntesis los errores estándar, estimados agrupando -cluster- a las empresas por la industria a la que pertenecen. Coeficientes estadísticamente significativos al: $1 \%+$, $5 \% \neq$ y $10 \%$ *

Fuente: Estimaciones propias del autor con base en datos de la Enterprise Survey del Banco Mundial, Estadísticas del Banco Mundial y Estadísticas Monetarias y Financieras del Fondo Monetario Internacional.

Al revisar los coeficientes de las diversas decisiones financieras, en el Cuadro 2 se muestra que el coeficiente del crédito comercial destaca sobre los coeficientes de las demás fuentes de financiamiento, resultado que corrobora lo hallado en las especificaciones del Cuadro 1. Los coeficientes de la variable que captura la presión competitiva, medida a través la introducción de nuevos productos, no son significativos estadísticamente e incluso no obtienen el signo esperado, mientras que los coeficientes de la segunda variable que intenta capturar las presiones competitivas en el mercado relacionadas con la mejora de procesos, sólo resultó ser ligeramente significativo estadísticamente, aunque con el signo contrario al esperado.

Con lo anterior, estos últimos resultados podrían estar reflejando que entre las empresas analizadas no se genera el efecto Darwiniano de la competencia. Es decir, que ante más competencia en el mercado, las empresas no mejorarán sus condiciones de producción, por lo que tendrían menos posibilidades de sobrevivir. Sin embargo, siguiendo lo sugerido por Boone (2008), una posible interpretación alternativa de los resultados obtenidos tiene que ver con el funcionamiento del mecanismo de competencia. Al respecto, Boone menciona que la competencia más intensa provoca el incremento en los beneficios de las empresas más eficientes respecto a las empresas menos eficientes. Por lo que, 
ante más competencia en el mercado se realiza una redistribución de los beneficios entre las empresas menos eficientes y las empresas más eficientes profit reallocation effect of competition-. Con ello, de acuerdo con los resultados del Cuadro 2 es posible que en la muestra de empresas considerada, el mecanis-mo competitivo propuesto por Boone no esté funcionando correctamente, ya que aún y cuando se introducen innovaciones en las condiciones de producción, con el fin de mejorar y diferenciarse de sus competidores, la mayor competencia en el mercado mantiene una relación negativa con el desempeño de las empresas. Por lo que la competencia en el mercado podría no estar propiciando la reasignación de beneficios entre las empresas menos eficientes y las más eficientes, y por lo tanto no generar una mejora en el desempeño de éstas.

Por su parte, los coeficientes para las dos características propias de cada empresa incluidas en las estimaciones son similares a los hallados en las especificaciones previas. El hecho de que las empresas sean exportadoras incide positivamente sobre el desempeño de las empresas, pero el coeficiente obtenido no es estadísticamente significativo. Mientras que la experiencia del administrador de la empresa, aún y cuando es estadísticamente significativa, no obtuvo el signo esperado, sugiriendo incluso que ante la mayor experiencia del administrador el desempeño de las empresas disminuye.

Como se mencionó, para controlar las estimaciones por diferencias en el grado de desarrollo de las economías, se estimaron algunas regresiones para tres subconjuntos de países, definidos éstos por el nivel del ingreso per-cápita en 2006, de acuerdo con datos del Banco Mundial. Un primer grupo (Grupo A) se integró por países cuyo ingreso per-cápita superó los 5.000 dólares estadounidenses, por lo que en éste se incluyen a: Argentina, Chile, México y Uruguay. Colombia y Panamá conforman un segundo conjunto de países (Grupo B), economías en las cuales el ingreso per-cápita se ubicó entre 3.000 y 5.000 dólares estadounidenses. Por último Bolivia, Ecuador, El Salvador, Guatemala, Honduras, Nicaragua, Paraguay y Perú componen el tercer grupo (Grupo C), cuyo ingreso per-cápita fue inferior a 3.000 dólares estadounidenses.

En el Cuadro 3 se muestran los resultados de estas estimaciones, los cuales muestran diferencias en los coeficientes de las diversas variables consideradas de acuerdo al grado de desarrollo de las economías en cuestión. Así, para el Grupo A el capital (activos fijos) es el insumo más importante en la determinación de las ventas de las empresas, hecho que contrasta con lo hallado para las empresas en economías cuyo nivel de desarrollo es más bajo -Grupo B y Grupo $\mathrm{C}$ - en donde el efecto del trabajo es superior incluso al coeficiente del capital para las empresas de los países del Grupo A. La variable sobre el acceso de las empresas al sistema financiero muestra también diferencias de acuerdo al grado de desarrollo de las economías. Puesto que mientras para las economías con 
mejores niveles de ingreso, si bien el coeficiente hallado es positivo, éste es inferior al hallado para las economías con un menor ingreso per-cápita. Este hecho podría estar sugiriendo que para las empresas en países con bajos niveles de desarrollo, el acceso a las fuentes de financiamiento afecta más su desempeño. Esto es consistente con el planteamiento sobre el desarrollo de los mercados financieros como un medio para la mejora de la productividad de las empresas, y con ello para la economía en su conjunto.

Respecto a las relaciones de las decisiones financieras sobre el desempeño de las empresas, los resultados del Cuadro 3 muestran que, genéricamente, para las empresas del Grupo A el empleo de recursos propios guarda una relación negativa, hecho que contrasta con los resultados hallados para las empresas en economías de menores ingresos -Grupo C-, en cuyo caso el coeficiente de esta variable en las diversas especificaciones es positivo y en la mayoría de éstas es estadísticamente significativo. ${ }^{15}$ Por otra parte, los resultados sugieren que sin importar el grado de desarrollo de las economías, el uso del crédito comercial representa sin duda una fuente de recursos importante para las empresas; ello se denota porque tanto para las empresas del Grupo A como para las empresas del Grupo C, esta variable es positiva y en la mayoría de las especificaciones es estadísticamente significativa. En este sentido, los resultados de este cuadro corroboran, parcialmente, lo propuesto por Fisman (2001) y Fisman y Love (2003) sobre el énfasis en el uso del crédito comercial como una de las principales fuentes de financiamiento de las empresas.

Los resultados de las estimaciones que incluyen los proxies de competencia construidos a partir del número de competidores enfrentados (Cuadro 3a; Cuadro 3c y Cuadro 3e), denotan nuevamente la existencia de una relación positiva entre un nivel "moderado" de la competencia y el desempeño de las empresas. Mientras que para los niveles "extremos" de competencia, esta relación es negativa. En este caso, los resultados no muestran grandes contrastes al considerar las diferencias en el grado de desarrollo de las economías. Por su parte, en las estimaciones que incluyen los proxies relacionados con las presiones competitivas, producto de los procesos de innovación -introducción o mejora de productos o procesos-, los resultados hallados no son estadísticamente significativos, e incluso en algunos casos resultaron ser negativos.

Por último, en los coeficientes de las demás variables de control incluidas en estas estimaciones -actividad exportadora, experiencia del administrador, cortes de energía y facilidades de apertura-, aún y cuando existen diferencias entre los resultados de las diferentes sub-muestras, en éstos, o bien se hallaron signos contrarios a los esperados, o no son estadísticamente significativos.

${ }^{15}$ Estos últimos resultados podrían asociarse con cierta evidencia que corrobora la teoría del pecking order. 
Cuadro 3

a) Grupo A: i) Desempeño, decisiones financieras y número de competidores

\begin{tabular}{|c|c|c|c|c|c|c|c|c|c|c|}
\hline & \multicolumn{2}{|l|}{ (1) } & \multicolumn{2}{|l|}{ (2) } & \multicolumn{2}{|l|}{ (3) } & \multicolumn{2}{|l|}{ (4) } & \multicolumn{2}{|c|}{ (5) } \\
\hline \multirow{2}{*}{ Activos fijos } & 0,619 & $\dagger$ & 0,649 & $\ddagger$ & 0,642 & $\ddagger$ & 0,647 & $t$ & 0,630 & $\dagger$ \\
\hline & $(0,175)$ & & $(0,201)$ & & $(0,207)$ & & $(0,183)$ & & $(0,173)$ & \\
\hline \multirow{2}{*}{$\begin{array}{l}\text { Trabajadores } \\
\text { permanentes }\end{array}$} & 0,343 & & 0,304 & & 0,309 & & 0,323 & & 0,340 & \\
\hline & $(0,192)$ & & $(0,214)$ & & $(0,221)$ & & $(0,200)$ & & $(0,190)$ & \\
\hline \multirow{2}{*}{ Actividad exportadora } & 0,422 & & 0,427 & & 0,446 & & 0,365 & & 0,383 & \\
\hline & $(0,279)$ & & $(0,279)$ & & $(0,271)$ & & $(0,279)$ & & $(0,280)$ & \\
\hline \multirow{2}{*}{$\begin{array}{l}\text { Experiencia } \\
\text { administrador }\end{array}$} & $-0,011$ & * & $-0,012$ & & $-0,011$ & * & $-0,012$ & ${ }^{*}$ & $-0,011$ & * \\
\hline & $(0,005)$ & & $(0,006)$ & & $(0,006)$ & & $(0,006)$ & & $(0,005)$ & \\
\hline \multirow{2}{*}{$\begin{array}{l}\text { Acceso al sistema } \\
\text { financiero }\end{array}$} & 0,303 & * & 0,311 & ${ }^{*}$ & 0,311 & * & 0,287 & ${ }^{*}$ & 0,288 & \\
\hline & $(0,160)$ & & $(0,14)$ & & $(0,143)$ & & $(0,151)$ & & $(0,162)$ & \\
\hline \multirow{2}{*}{$\begin{array}{l}\text { Facilidades de } \\
\text { apertura }\end{array}$} & 0,079 & $\ddagger$ & 0,067 & $\ddagger$ & 0,071 & $\ddagger$ & 0,075 & $\ddagger$ & 0,081 & $\ddagger$ \\
\hline & $(0,026)$ & & $(0,022)$ & & $(0,022)$ & & $(0,023)$ & & $(0,027)$ & \\
\hline \multirow{2}{*}{ Cortes de energía } & 0,116 & & 0,117 & & 0,108 & & 0,135 & & 0,128 & \\
\hline & $(0,151)$ & & $(0,153)$ & & $(0,155)$ & & $(0,143)$ & & $(0,144)$ & \\
\hline \multirow{2}{*}{$\begin{array}{l}\text { Uso de recursos } \\
\text { propios }\end{array}$} & $-0,133$ & & $-0,131$ & & $-0,129$ & & $-0,149$ & ${ }^{*}$ & $-0,145$ & * \\
\hline & $(0,075)$ & & $(0,083)$ & & $(0,082)$ & & $(0,075)$ & & $(0,072)$ & \\
\hline \multirow{2}{*}{$\begin{array}{l}\text { Uso de recursos } \\
\text { bancarios }\end{array}$} & 0,235 & * & 0,250 & ${ }^{*}$ & 0,255 & * & 0,224 & * & 0,223 & * \\
\hline & $(0,108)$ & & $(0,121)$ & & $(0,122)$ & & $(0,104)$ & & $(0,104)$ & \\
\hline \multirow{2}{*}{$\begin{array}{l}\text { Uso de crédito } \\
\text { comercial }\end{array}$} & 0,401 & * & 0,393 & ${ }^{*}$ & 0,411 & * & 0,379 & ${ }^{*}$ & 0,393 & * \\
\hline & $(0,175)$ & & $(0,172)$ & & $(0,181)$ & & $(0,166)$ & & $(0,172)$ & \\
\hline \multirow{2}{*}{ Uso de otros recursos } & $-0,061$ & & $-0,074$ & & $-0,091$ & & $-0,047$ & & $-0,050$ & \\
\hline & $(0,129)$ & & $(0,154)$ & & $(0,136)$ & & $(0,132)$ & & $(0,119)$ & \\
\hline \multirow{2}{*}{ Competencia (total) } & $-0,085$ & ${ }^{*}$ & \multirow{2}{*}{\multicolumn{2}{|c|}{-}} & \multirow{2}{*}{\multicolumn{2}{|c|}{-}} & \multirow{2}{*}{\multicolumn{2}{|c|}{-}} & \multirow{2}{*}{\multicolumn{2}{|c|}{-}} \\
\hline & $(0,040)$ & & & & & & & & & \\
\hline \multirow{2}{*}{ Sin competencia } & \multirow{2}{*}{\multicolumn{2}{|c|}{-}} & $-0,135$ & & \multirow{2}{*}{\multicolumn{2}{|c|}{ - }} & \multirow{2}{*}{\multicolumn{2}{|c|}{-}} & - & \\
\hline & & & $(0,175)$ & & & & & & & \\
\hline 1 comnetider & $\ldots$ & & $\ldots$ & & 0,487 & * & $\ldots$ & & & \\
\hline T competiaor & - & & & & $(0,243)$ & & - & & - & \\
\hline Entre 2 y 5 & - & & - & & - & & 0,301 & $\ddagger$ & - & \\
\hline competidores & & & & & & & $(0,123)$ & & & \\
\hline Más de 5 & - & & - & & $\ldots$ & & $\ldots$ & & $-0,327$ & $\ddagger$ \\
\hline competidores & - & & - & & - & & - & & $(0,128)$ & \\
\hline Constante & $-0,603$ & & $-0,551$ & & $-0,713$ & & $-0,940$ & & $-0,839$ & \\
\hline Constante & $(1,218)$ & & $(1,093)$ & & $(1,078)$ & & $(1,156)$ & & $(1,242)$ & \\
\hline Observaciones & 1.428 & & 1.428 & & 1.428 & & 1.428 & & 1.428 & \\
\hline R2 (ajustada) & 0,645 & & 0,624 & & 0,630 & & 0,632 & & 0,643 & \\
\hline
\end{tabular}


Cuadro 3 (continuación)

b) Grupo A: ii) Desempeño, decisiones financieras e innovación y competencia

\begin{tabular}{|c|c|c|c|c|c|c|c|c|}
\hline & (1) & & (2) & & (3) & & (4) & \\
\hline \multirow{2}{*}{ Activos fijos } & 0,729 & $\dagger$ & 0,697 & $\dagger$ & 0,667 & $\dagger$ & 0,681 & $\dagger$ \\
\hline & $(0,151)$ & & $(0,167)$ & & $(0,164)$ & & $(0,166)$ & \\
\hline \multirow{2}{*}{$\begin{array}{l}\text { Trabajadores } \\
\text { permanentes }\end{array}$} & 0,192 & & 0,233 & & 0,261 & & 0,261 & \\
\hline & $(0,224)$ & & $(0,199)$ & & $(0,214)$ & & $(0,189)$ & \\
\hline \multirow{2}{*}{ Actividad exportadora } & 0,367 & & 0,370 & & 0,402 & & 0,401 & \\
\hline & $(0,265)$ & & $(0,266)$ & & $(0,248)$ & & $(0,295)$ & \\
\hline \multirow{2}{*}{ Experiencia administrador } & $-0,012$ & & $-0,012$ & * & $-0,013$ & * & $-0,011$ & * \\
\hline & $(0,007)$ & & $(0,006)$ & & $(0,006)$ & & $(0,006)$ & \\
\hline \multirow{2}{*}{$\begin{array}{l}\text { Acceso al sistema } \\
\text { financiero }\end{array}$} & 0,342 & * & 0,313 & & 0,331 & * & 0,315 & * \\
\hline & $(0,153)$ & & $(0,147)$ & & $(0,166)$ & & $(0,145)$ & \\
\hline \multirow{2}{*}{ Facilidades de apertura } & 0,052 & $\ddagger$ & 0,062 & $\dagger$ & 0,062 & $\ddagger$ & 0,070 & $\dagger$ \\
\hline & $(0,021)$ & & $(0,016)$ & & $(0,026)$ & & $(0,016)$ & \\
\hline \multirow{2}{*}{ Cortes de energía } & 0,127 & & 0,132 & & 0,114 & & 0,126 & \\
\hline & $(0,121)$ & & $(0,129)$ & & $(0,153)$ & & $(0,128)$ & \\
\hline \multirow{2}{*}{ Uso de recursos propios } & $-0,171$ & $\ddagger$ & $-0,188$ & $\ddagger$ & $-0,116$ & & $-0,194$ & $\ddagger$ \\
\hline & $(0,065)$ & & $(0,063)$ & & $(0,069)$ & & $(0,070)$ & \\
\hline \multirow{2}{*}{ Uso de recursos bancarios } & 0,142 & & 0,160 & & 0,201 & & 0,182 & \\
\hline & $(0,139)$ & & $(0,115)$ & & $(0,133)$ & & $(0,123)$ & \\
\hline \multirow{2}{*}{ Uso de crédito comercial } & 0,386 & * & 0,369 & * & 0,423 & $\ddagger$ & 0,368 & * \\
\hline & $(0,177)$ & & $(0,167)$ & & $(0,174)$ & & $(0,170)$ & \\
\hline \multirow{2}{*}{ Uso de otros recursos } & $-0,088$ & & $-0,107$ & & $-0,057$ & & $-0,101$ & \\
\hline & $(0,151)$ & & $(0,168)$ & & $(0,130)$ & & $(0,154)$ & \\
\hline \multirow{2}{*}{ Competencia (total) } & \multirow{2}{*}{\multicolumn{2}{|c|}{-}} & \multirow{2}{*}{\multicolumn{2}{|c|}{-}} & $-0,089$ & * & \multirow{2}{*}{\multicolumn{2}{|c|}{ - }} \\
\hline & & & & & $(0,044)$ & & & \\
\hline \multirow{2}{*}{$\begin{array}{l}\text { Introducción o mejora de } \\
\text { producto }\end{array}$} & 0,296 & & \multirow{2}{*}{\multicolumn{2}{|c|}{ - }} & 0,197 & & \multirow{2}{*}{\multicolumn{2}{|c|}{-}} \\
\hline & $(0,239)$ & & & & $(0,204)$ & & & \\
\hline \multirow{2}{*}{ Mejora de procesos } & \multirow{2}{*}{\multicolumn{2}{|c|}{-}} & 0,216 & & 0,122 & & \multirow{2}{*}{\multicolumn{2}{|c|}{ - }} \\
\hline & & & $(0,181)$ & & $(0,102)$ & & & \\
\hline \multirow{2}{*}{ Constante } & $-0,174$ & & $-0,409$ & & $-0,099$ & & $-0,659$ & \\
\hline & $(1,292)$ & & $(1,103)$ & & $(1,364)$ & & $(1,050)$ & \\
\hline Observaciones & 1.570 & & 1.572 & & 1.424 & & 1.573 & \\
\hline R2 (ajustada) & 0,590 & & 0,608 & & 0,616 & & 0,618 & \\
\hline
\end{tabular}


Cuadro 3 (continuación)

c) Grupo B: i) Desempeño, decisiones financieras y número de competidores

\begin{tabular}{|c|c|c|c|c|c|c|c|c|c|c|}
\hline & (1) & & (2) & & (3) & & (4) & & (5) & \\
\hline \multirow{2}{*}{ Activos fijos } & $-0,133$ & & $-0,198$ & & $-0,177$ & & $-0,216$ & & $-0,134$ & \\
\hline & $(0,201)$ & & $(0,285)$ & & $(0,260)$ & & $(0,265)$ & & $(0,211)$ & \\
\hline \multirow{2}{*}{$\begin{array}{l}\text { Trabajadores } \\
\text { permanentes }\end{array}$} & 1,741 & $\dagger$ & 1,833 & $\dagger$ & 1,795 & $\dagger$ & 1,867 & $\dagger$ & 1,749 & $\dagger$ \\
\hline & $(0,327)$ & & $(0,455)$ & & $(0,428)$ & & $(0,419)$ & & $(0,331)$ & \\
\hline \multirow{2}{*}{ Actividad exportadora } & $-0,48$ & & $-0,56$ & & $-0,52$ & & $-0,61$ & & $-0,5$ & \\
\hline & $(0,419)$ & & $(0,546)$ & & $(0,525)$ & & $(0,522)$ & & $(0,417)$ & \\
\hline \multirow{2}{*}{$\begin{array}{l}\text { Experiencia } \\
\text { administrador }\end{array}$} & $-0,033$ & $\dagger$ & $-0,032$ & $\dagger$ & $-0,033$ & $\dagger$ & $-0,032$ & $\dagger$ & $-0,033$ & $\dagger$ \\
\hline & $(0,007)$ & & $(0,008)$ & & $(0,008)$ & & $(0,009)$ & & $(0,008)$ & \\
\hline \multirow{2}{*}{$\begin{array}{l}\text { Acceso al sistema } \\
\text { financiero }\end{array}$} & 0,117 & & 0,070 & & 0,078 & & 0,052 & & 0,116 & \\
\hline & $(0,235)$ & & $(0,305)$ & & $(0,307)$ & & $(0,296)$ & & $(0,232)$ & \\
\hline \multirow{2}{*}{$\begin{array}{l}\text { Facilidades de } \\
\text { apertura }\end{array}$} & $-0,046$ & $\dagger$ & $-0,052$ & $\ddagger$ & $-0,050$ & $\ddagger$ & $-0,049$ & $\dagger$ & $-0,045$ & $\dagger$ \\
\hline & $(0,01)$ & & $(0,017)$ & & $(0,016)$ & & $(0,013)$ & & $(0,010)$ & \\
\hline \multirow{2}{*}{ Cortes de energía } & $-0,03$ & & $-0,04$ & & $-0,05$ & & $-0,03$ & & $-0,02$ & \\
\hline & $(0,028)$ & & $(0,044)$ & & $(0,049)$ & & $(0,030)$ & & $(0,025)$ & \\
\hline \multirow{2}{*}{$\begin{array}{l}\text { Uso de recursos } \\
\text { propios }\end{array}$} & 0,140 & $\ddagger$ & 0,149 & $\ddagger$ & 0,150 & $\ddagger$ & 0,134 & * & 0,132 & * \\
\hline & $(0,055)$ & & $(0,047)$ & & $(0,049)$ & & $(0,066)$ & & $(0,061)$ & \\
\hline \multirow{2}{*}{$\begin{array}{l}\text { Uso de recursos } \\
\text { bancarios }\end{array}$} & 0,835 & & 0,914 & & 0,886 & & 0,920 & * & 0,831 & * \\
\hline & $(0,393)$ & & $(0,520)$ & & $(0,505)$ & & $(0,462)$ & & $(0,383)$ & \\
\hline \multirow{2}{*}{$\begin{array}{l}\text { Uso de crédito } \\
\text { comercial }\end{array}$} & 0,193 & & 0,184 & & 0,187 & & 0,157 & & 0,184 & \\
\hline & $(0,11)$ & & $(0,122)$ & & $(0,115)$ & & $(0,102)$ & & $(0,103)$ & \\
\hline \multirow{2}{*}{$\begin{array}{l}\text { Uso de otros } \\
\text { recursos }\end{array}$} & $-0,140$ & & $-0,179$ & & $-0,171$ & & $-0,203$ & & $-0,145$ & \\
\hline & $(0,181)$ & & $(0,183)$ & & $(0,164)$ & & $(0,132)$ & & $(0,189)$ & \\
\hline \multirow{2}{*}{ Competencia (total) } & $-0,004$ & & \multirow{2}{*}{\multicolumn{2}{|c|}{ - }} & \multirow{2}{*}{\multicolumn{2}{|c|}{ - }} & \multirow{2}{*}{\multicolumn{2}{|c|}{ - }} & \multirow{2}{*}{\multicolumn{2}{|c|}{ - }} \\
\hline & $(0,062)$ & & & & & & & & & \\
\hline \multirow{2}{*}{ Sin competencia } & \multirow{2}{*}{\multicolumn{2}{|c|}{-}} & $-0,399$ & & \multirow{2}{*}{\multicolumn{2}{|c|}{ - }} & \multirow{2}{*}{\multicolumn{2}{|c|}{ - }} & \multirow{2}{*}{\multicolumn{2}{|c|}{ - }} \\
\hline & & & $(0,313)$ & & & & & & & \\
\hline \multirow{2}{*}{1 competidor } & \multirow{2}{*}{\multicolumn{2}{|c|}{-}} & \multirow{2}{*}{\multicolumn{2}{|c|}{-}} & $-0,352$ & * & \multirow{2}{*}{\multicolumn{2}{|c|}{-}} & 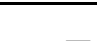 & \\
\hline & & & & & $(0,169)$ & & & & - & \\
\hline Entre 2 y 5 & - & & - & & - & & 0,289 & & 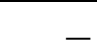 & \\
\hline competidores & & & & & & & $(0,404)$ & & - & \\
\hline Más de 5 & $\ldots$ & & - & & - & & - & & $-0,119$ & \\
\hline competidores & & & & & & & & & $(0,24)$ & \\
\hline Constante & 3,884 & $\dagger$ & 3,900 & $\dagger$ & 2,917 & $\dagger$ & 3,617 & $\dagger$ & 3,861 & $\dagger$ \\
\hline constante & $(0,138)$ & & $(0,245)$ & & $(0,208)$ & & $(0,486)$ & & $(0,226)$ & \\
\hline Observaciones & 549 & & 549 & & 549 & & 549 & & 549 & \\
\hline R2 (ajustada) & 0,771 & & 0,751 & & 0,759 & & 0,745 & & 0,770 & \\
\hline
\end{tabular}


Cuadro 3 (continuación)

d) Grupo B: ii) Desempeño, decisiones financieras e innovación y competencia

\begin{tabular}{|c|c|c|c|c|c|c|c|c|}
\hline & \multicolumn{2}{|l|}{ (1) } & \multicolumn{2}{|l|}{ (2) } & \multicolumn{2}{|l|}{ (3) } & \multicolumn{2}{|c|}{ (4) } \\
\hline \multirow{2}{*}{ Activos fijos } & $-0,313$ & & 0,052 & & $-0,227$ & & $-0,207$ & \\
\hline & $(0,520)$ & & $(0,316)$ & & $(0,237)$ & & $(0,340)$ & \\
\hline \multirow{2}{*}{$\begin{array}{l}\text { Trabajadores } \\
\text { permanentes }\end{array}$} & 1,742 & * & 1,404 & $\ddagger$ & 1,678 & $\dagger$ & 1,771 & $\ddagger$ \\
\hline & $(0,742)$ & & $(0,501)$ & & $(0,357)$ & & $(0,543)$ & \\
\hline \multirow{2}{*}{ Actividad exportadora } & $-0,310$ & & $-0,173$ & & $-0,324$ & & $-0,457$ & \\
\hline & $(0,675)$ & & $(0,464)$ & & $(0,393)$ & & $(0,564)$ & \\
\hline \multirow{2}{*}{ Experiencia administrador } & $-0,020$ & * & $-0,031$ & $\dagger$ & $-0,024$ & $\dagger$ & $-0,029$ & $\dagger$ \\
\hline & $(0,009)$ & & $(0,006)$ & & $(0,006)$ & & $(0,007)$ & \\
\hline \multirow{2}{*}{$\begin{array}{l}\text { Acceso al sistema } \\
\text { financiero }\end{array}$} & 0,199 & & 0,358 & & 0,093 & & 0,180 & \\
\hline & $(0,422)$ & & $(0,208)$ & & $(0,317)$ & & $(0,262)$ & \\
\hline \multirow{2}{*}{ Facilidades de apertura } & $-0,047$ & & $-0,038$ & * & $-0,044$ & $\dagger$ & $-0,047$ & $\ddagger$ \\
\hline & $(0,024)$ & & $(0,016)$ & & $(0,008)$ & & $(0,019)$ & \\
\hline \multirow{2}{*}{ Cortes de energía } & $-0,074$ & & $-0,034$ & & 0,055 & & $-0,079$ & \\
\hline & $(0,236)$ & & $(0,107)$ & & $(0,049)$ & & $(0,171)$ & \\
\hline \multirow{2}{*}{ Uso de recursos propios } & 0,193 & $\dagger$ & 0,117 & * & 0,167 & * & 0,144 & $\dagger$ \\
\hline & $(0,031)$ & & $(0,058)$ & & $(0,073)$ & & $(0,036)$ & \\
\hline \multirow{2}{*}{ Uso de recursos bancarios } & 0,913 & & 0,586 & & 0,897 & * & 0,842 & \\
\hline & $(0,687)$ & & $(0,441)$ & & $(0,398)$ & & $(0,533)$ & \\
\hline \multirow{2}{*}{ Uso de crédito comercial } & 0,412 & & 0,276 & * & 0,343 & & 0,227 & \\
\hline & $(0,285)$ & & $(0,131)$ & & $(0,195)$ & & $(0,169)$ & \\
\hline \multirow{2}{*}{ Uso de otros recursos } & $-0,403$ & & $-0,135$ & & $-0,255$ & & $-0,254$ & \\
\hline & $(0,414)$ & & $(0,140)$ & & $(0,150)$ & & $(0,184)$ & \\
\hline \multirow{2}{*}{ Competencia (total) } & \multirow{2}{*}{\multicolumn{2}{|c|}{-}} & \multirow{2}{*}{\multicolumn{2}{|c|}{-}} & $-0,038$ & & \multirow{2}{*}{\multicolumn{2}{|c|}{ - }} \\
\hline & & & & & $(0,067)$ & & & \\
\hline \multirow{2}{*}{$\begin{array}{l}\text { Introducción o mejora de } \\
\text { producto }\end{array}$} & $-0,559$ & $\ddagger$ & \multirow{2}{*}{\multicolumn{2}{|c|}{ - }} & $-0,633$ & $\ddagger$ & \multirow{2}{*}{\multicolumn{2}{|c|}{ - }} \\
\hline & $(0,178)$ & & & & $(0,177)$ & & & \\
\hline \multirow{2}{*}{ Mejora de procesos } & \multirow{2}{*}{\multicolumn{2}{|c|}{-}} & $-0,198$ & & 0,236 & & \multirow{2}{*}{\multicolumn{2}{|c|}{ - }} \\
\hline & & & $(0,111)$ & & $(0,137)$ & & & \\
\hline \multirow{2}{*}{ Constante } & 4,500 & $t$ & 4,056 & $\dagger$ & 4,586 & $\dagger$ & 3,995 & $\dagger$ \\
\hline & $(0,486)$ & & $(0,204)$ & & $(0,398)$ & & $(0,257)$ & \\
\hline Observaciones & 589 & & 590 & & 548 & & 590 & \\
\hline R2 (ajustada) & 0,755 & & 0,821 & & 0,784 & & 0,758 & \\
\hline
\end{tabular}


Cuadro 3 (continuación)

e) Grupo C: i) Desempeño, decisiones financieras y número de competidores

\begin{tabular}{|c|c|c|c|c|c|c|c|c|c|c|}
\hline & \multicolumn{2}{|c|}{ (1) } & \multicolumn{2}{|l|}{ (2) } & \multicolumn{2}{|c|}{ (3) } & \multicolumn{2}{|l|}{ (4) } & \multicolumn{2}{|c|}{ (5) } \\
\hline \multirow{2}{*}{ Activos fijos } & $-0,109$ & & $-0,104$ & & $-0,106$ & & $-0,119$ & & $-0,116$ & \\
\hline & $(0,085)$ & & $(0,082)$ & & $(0,082)$ & & $(0,096)$ & & $(0,092)$ & \\
\hline \multirow{2}{*}{$\begin{array}{l}\text { Trabajadores } \\
\text { permanentes }\end{array}$} & 1,332 & $\dagger$ & 1,315 & $\dagger$ & 1,32 & $t$ & 1,34 & $\dagger$ & 1,342 & $\dagger$ \\
\hline & $(0,122)$ & & $(0,115)$ & & $(0,117)$ & & $(0,126)$ & & $(0,126)$ & \\
\hline \multirow{2}{*}{ Actividad exportadora } & 0,177 & & 0,185 & & 0,181 & & 0,188 & & 0,181 & \\
\hline & $(0,129)$ & & $(0,125)$ & & $(0,128)$ & & $(0,117)$ & & $(0,123)$ & \\
\hline \multirow{2}{*}{$\begin{array}{l}\text { Experiencia } \\
\text { administrador }\end{array}$} & 0,006 & * & 0,006 & $\ddagger$ & 0,006 & $\ddagger$ & 0,006 & ${ }^{*}$ & 0,005 & ${ }^{*}$ \\
\hline & $(0,003)$ & & $(0,003)$ & & $(0,003)$ & & $(0,003)$ & & $(0,003)$ & \\
\hline \multirow{2}{*}{$\begin{array}{l}\text { Acceso al sistema } \\
\text { financiero }\end{array}$} & 0,438 & $\dagger$ & 0,459 & $\dagger$ & 0,456 & $\dagger$ & 0,431 & $\dagger$ & 0,427 & $\dagger$ \\
\hline & $(0,057)$ & & $(0,054)$ & & $(0,053)$ & & $(0,051)$ & & $(0,056)$ & \\
\hline \multirow{2}{*}{$\begin{array}{l}\text { Facilidades de } \\
\text { apertura }\end{array}$} & 0,033 & $\dagger$ & 0,032 & $\dagger$ & 0,032 & $t$ & 0,034 & $\dagger$ & 0,033 & $\dagger$ \\
\hline & $(0,003)$ & & $(0,003)$ & & $(0,003)$ & & $(0,003)$ & & $(0,003)$ & \\
\hline \multirow{2}{*}{ Cortes de energía } & $-0,01$ & & $-0,03$ & & $-0,02$ & & $-0,02$ & & $-0,01$ & \\
\hline & $(0,104)$ & & $(0,093)$ & & $(0,096)$ & & $(0,102)$ & & $(0,107)$ & \\
\hline \multirow{2}{*}{$\begin{array}{l}\text { Uso de recursos } \\
\text { propios }\end{array}$} & 0,234 & $\dagger$ & 0,252 & $\dagger$ & 0,250 & $\dagger$ & 0,217 & $\dagger$ & 0,218 & $\dagger$ \\
\hline & $(0,055)$ & & $(0,055)$ & & $(0,054)$ & & $(0,051)$ & & $(0,053)$ & \\
\hline \multirow{2}{*}{$\begin{array}{l}\text { Uso de recursos } \\
\text { bancarios }\end{array}$} & $-0,114$ & & $-0,102$ & & $-0,103$ & & $-0,128$ & & $-0,126$ & \\
\hline & $(0,080)$ & & $(0,083)$ & & $(0,082)$ & & $(0,078)$ & & $(0,079)$ & \\
\hline \multirow{2}{*}{$\begin{array}{l}\text { Uso de crédito } \\
\text { comercial }\end{array}$} & 0,226 & $\ddagger$ & 0,227 & * & 0,230 & $\ddagger$ & 0,205 & * & 0,214 & $\ddagger$ \\
\hline & $(0,095)$ & & $(0,102)$ & & $(0,101)$ & & $(0,097)$ & & $(0,093)$ & \\
\hline \multirow{2}{*}{ Uso de otros recursos } & $-0,170$ & & $-0,185$ & & $-0,182$ & & $-0,174$ & & $-0,165$ & \\
\hline & $(0,154)$ & & $(0,159)$ & & $(0,157)$ & & $(0,132)$ & & $(0,159)$ & \\
\hline \multirow{2}{*}{ Competencia (total) } & $-0,054$ & $\ddagger$ & \multirow{2}{*}{\multicolumn{2}{|c|}{ - }} & \multirow{2}{*}{\multicolumn{2}{|c|}{-}} & \multirow{2}{*}{\multicolumn{2}{|c|}{-}} & \multirow{2}{*}{\multicolumn{2}{|c|}{ - }} \\
\hline & $(0,017)$ & & & & & & & & & \\
\hline \multirow{2}{*}{ Sin competencia } & \multirow{2}{*}{\multicolumn{2}{|c|}{ - }} & $-0,130$ & $\ddagger$ & \multirow{2}{*}{\multicolumn{2}{|c|}{ - }} & \multirow{2}{*}{\multicolumn{2}{|c|}{ - }} & - & \\
\hline & & & $(0,055)$ & & & & & & & \\
\hline 1 comnetidor & - & & - & & 0,045 & & - & & - & \\
\hline ' competiuer & - & & - & & $(0,24)$ & & - & & - & \\
\hline Entre 2 y 5 & - & & - & & - & & 0,263 & $\dagger$ & - & \\
\hline competidores & & & & & & & $(0,026)$ & & & \\
\hline Más de 5 & - & & - & & - & & - & & $-0,237$ & $\dagger$ \\
\hline competidores & & & & & & & & & $(0,042)$ & \\
\hline Constante & $-2,054$ & $\dagger$ & $-0,343$ & & $-0,355$ & & $-2,407$ & $\dagger$ & $-0,404$ & \\
\hline 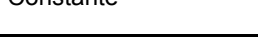 & $(0,336)$ & & $(0,443)$ & & $(0,459)$ & & $(0,346)$ & & $(0,477)$ & \\
\hline Observaciones & 1,543 & & 1,543 & & 1,543 & & 1,543 & & 1,543 & \\
\hline R2 (ajustada) & 0,624 & & 0,625 & & 0,624 & & 0,623 & & 0,623 & \\
\hline
\end{tabular}


Cuadro 3 (continuación)

f) Grupo C: ii) Desempeño, decisiones financieras e innovación y competencia

\begin{tabular}{|c|c|c|c|c|c|}
\hline & (1) & (2) & (3) & (4) & \\
\hline Activos fijos & $\begin{array}{r}-0,156 \\
(0,109) \\
\end{array}$ & $\begin{array}{r}-0,153 \\
(0,100) \\
\end{array}$ & $\begin{array}{l}-0,111 \\
(0,086)\end{array}$ & $\begin{array}{r}-0,155 \\
(0,101)\end{array}$ & \\
\hline $\begin{array}{l}\text { Trabajadores } \\
\text { permanentes }\end{array}$ & $\begin{array}{r}1,234 \\
(0,195)\end{array}$ & $\begin{array}{r}1,231 \\
(0,181)\end{array}$ & $\begin{array}{r}1,349 \\
(0,134)\end{array}$ & $\begin{array}{r}1,229 \\
(0,179)\end{array}$ & $\dagger$ \\
\hline Actividad exportadora & $\begin{array}{r}0,338 \quad \ddagger \\
(0,118)\end{array}$ & $\begin{array}{r}0,329 \\
(0,124)\end{array}$ & $\begin{array}{r}0,189 \\
(0,130)\end{array}$ & $\begin{array}{r}0,327 \\
(0,118)\end{array}$ & $\ddagger$ \\
\hline Experiencia administrador & $\begin{array}{rr}0,006 & \ddagger \\
(0,003) & \end{array}$ & $\begin{array}{rr}0,006 & \ddagger \\
(0,003) & \end{array}$ & $\begin{array}{r}0,005 \\
(0,003) \\
\end{array}$ & $\begin{array}{r}0,006 \\
(0,003) \\
\end{array}$ & $\ddagger$ \\
\hline $\begin{array}{l}\text { Acceso al sistema } \\
\text { financiero }\end{array}$ & $\begin{array}{r}0,493 \quad \dagger \\
(0,039)\end{array}$ & $\begin{array}{r}0,485 \\
(0,037)\end{array}$ & $\begin{array}{r}0,451 \\
(0,057)\end{array}$ & $\begin{array}{r}0,483 \\
(0,039)\end{array}$ & $\dagger$ \\
\hline Facilidades de apertura & $\begin{array}{r}0,037 \\
(0,004)\end{array}$ & $\begin{array}{r}0,037 \\
(0,004)\end{array}$ & $\begin{array}{r}0,033 \quad \dagger \\
(0,003)\end{array}$ & $\begin{array}{r}0,037 \\
(0,004)\end{array}$ & $\dagger$ \\
\hline Cortes de energía & $\begin{array}{r}-0,080 \\
(0,096)\end{array}$ & $\begin{array}{r}-0,076 \\
(0,100)\end{array}$ & $\begin{array}{r}-0,013 \\
(0,097) \\
\end{array}$ & $\begin{array}{r}-0,078 \\
(0,1) \\
\end{array}$ & \\
\hline Uso de recursos propios & $\begin{array}{r}0,154 \text { * } \\
(0,074)\end{array}$ & $\begin{array}{l}0,150 \quad \text { * } \\
(0,07)\end{array}$ & $\begin{array}{rr}0,236 & \dagger \\
(0,062) & \\
\end{array}$ & $\begin{array}{r}0,152 \\
(0,071) \\
\end{array}$ & * \\
\hline Uso de recursos bancarios & $\begin{array}{r}0,007 \\
(0,097) \\
\end{array}$ & $\begin{array}{r}0,002 \\
(0,095) \\
\end{array}$ & $\begin{array}{r}-0,104 \\
(0,078) \\
\end{array}$ & $\begin{array}{r}-0,001 \\
(0,100) \\
\end{array}$ & \\
\hline Uso de crédito comercial & $\begin{array}{r}0,230 \text { * } \\
(0,109)\end{array}$ & $\begin{array}{r}0,234 \text { * } \\
(0,107)\end{array}$ & $\begin{array}{r}0,226 \\
(0,105) \\
\end{array}$ & $\begin{array}{r}0,232 \\
(0,104) \\
\end{array}$ & * \\
\hline Uso de otros recursos & $\begin{array}{r}-0,218 \\
(0,138) \\
\end{array}$ & $\begin{array}{r}-0,233 \\
(0,143) \\
\end{array}$ & $\begin{array}{r}-0,162 \\
(0,135) \\
\end{array}$ & $\begin{array}{r}-0,229 \\
(0,148) \\
\end{array}$ & \\
\hline Competencia (total) & - & - & $\begin{array}{cc}-0,052 & \dagger \\
(0,016) & \\
\end{array}$ & - & \\
\hline $\begin{array}{l}\text { Introducción o mejora de } \\
\text { producto }\end{array}$ & $\begin{array}{l}-0,120 \\
(0,152)\end{array}$ & 一 & $\begin{array}{l}-0,159 \\
(0,164)\end{array}$ & - & \\
\hline Mejora de procesos & - & $\begin{array}{r}-0,059 \\
(0,074)\end{array}$ & $\begin{array}{r}-0,047 \\
(0,101)\end{array}$ & - & \\
\hline Constante & $\begin{array}{r}-0,275 \\
(0,543) \\
\end{array}$ & $\begin{array}{r}-0,325 \\
(0,563) \\
\end{array}$ & $\begin{array}{r}-0,120 \\
(0,449) \\
\end{array}$ & $\begin{array}{r}-2,153 \\
(0,466) \\
\end{array}$ & $\dagger$ \\
\hline Observaciones & 1.767 & 1.764 & 1.540 & 1.767 & \\
\hline R2 (ajustada) & 0,622 & 0,623 & 0,624 & 0,622 & \\
\hline
\end{tabular}

Notas: Estimaciones utilizando el método de mínimos cuadrados en dos etapas. Las variables instrumentadas fueron las referentes a los activos fijos (cap) y los trabajadores permanentes (tra). En todas las especificaciones se incluyen como variables independientes el crecimiento del PIB y la inflación, se incluyen también dummies por industria. Se reportan entre paréntesis los errores estándar, estimados agrupando cluster- a las empresas por la industria a la que pertenecen. Coeficientes estadísticamente significativos al: $1 \%$ †, 5\% y 10\%*. Grupo A: Argentina, Chile, México y Uruguay (ingreso per-cápita superior a 5.000 dólares norteamericanos). Grupo B: Colombia y Panamá (ingreso per-cápita entre 3.000 y 5.000 dólares norteamericanos). Grupo C: Bolivia, Ecuador, El Salvador, Guatemala, Honduras, Nicaragua, Paraguay y Perú (ingreso per-cápita inferior a 3.000 dólares norteamericanos).

Fuente: Estimaciones propias del autor con base en datos de la Enterprise Survey del Banco Mundial, Estadísticas del Banco Mundial y Estadísticas Monetarias y Financieras del Fondo Monetario Internacional. 


\subsection{Nivel de ventas ante diferentes presiones competitivas}

Como se mencionó al inicio de esta sección, un segundo conjunto de ejercicios consistió en considerar diferentes muestras, definidas éstas según el grado de las presiones competitivas percibidas en el mercado. En el Cuadro 4 se resumen los resultados de las estimaciones de las muestras que consideraron en su construcción el número de competidores en el mercado. Mientras que el Cuadro 5 se muestran los resultados de las especificaciones con las muestras construidas a partir de las respuestas a la introducción o mejora de productos, y a la mejora de procesos, reflejando de esta manera la percepción de las empresas respecto a la presión competitiva propiciada por los procesos de innovación.

\section{Cuadro 4}

Desempeño, decisiones financieras y número de competidores ante diferente grado de competencia

\begin{tabular}{|c|c|c|c|c|c|c|c|c|}
\hline \multirow[b]{2}{*}{ Activos fijos } & \multicolumn{2}{|c|}{$\begin{array}{c}\text { Sin } \\
\text { competidores }\end{array}$} & \multicolumn{2}{|c|}{$\begin{array}{c}\text { Con un } \\
\text { competidor }\end{array}$} & \multicolumn{2}{|c|}{$\begin{array}{c}\text { Entre } 2 \text { y } 5 \\
\text { competidores }\end{array}$} & \multicolumn{2}{|c|}{$\begin{array}{c}\text { Más de } 5 \\
\text { competidores }\end{array}$} \\
\hline & $\begin{array}{c}0,202 \\
(0,086)\end{array}$ & * & $\begin{array}{c}0,244 \\
(0,139) \\
\end{array}$ & & $\begin{array}{c}0,112 \\
(0,140)\end{array}$ & & $\begin{array}{c}0,331 \\
(0,517)\end{array}$ & \\
\hline Trabajadores permanentes & $\begin{array}{c}0,943 \\
(0,162)\end{array}$ & $\dagger$ & $\begin{array}{c}1,203 \\
(0,342)\end{array}$ & $\dagger$ & $\begin{array}{c}0,904 \\
(0,495)\end{array}$ & * & $\begin{array}{c}0,882 \\
(0,676)\end{array}$ & \\
\hline Actividad exportadora & $\begin{array}{c}0,530 \\
(0,384)\end{array}$ & & $\begin{array}{c}-0,816 \\
(0,260)\end{array}$ & $\ddagger$ & $\begin{array}{c}0,528 \\
(0,394)\end{array}$ & & $\begin{array}{c}0,049 \\
(0,382)\end{array}$ & \\
\hline Experiencia administrador & $\begin{array}{c}0,003 \\
(0,008)\end{array}$ & & $\begin{array}{c}0,015 \\
(0,011) \\
\end{array}$ & & $\begin{array}{l}-0,002 \\
(0,005) \\
\end{array}$ & & $\begin{array}{l}-0,017 \\
(0,007) \\
\end{array}$ & $\ddagger$ \\
\hline Acceso al sistema financiero & $\begin{array}{c}-0,088 \\
(0,185) \\
\end{array}$ & & $\begin{array}{c}0,284 \\
(0,280) \\
\end{array}$ & & $\begin{array}{c}0,238 \\
(0,138) \\
\end{array}$ & & $\begin{array}{c}0,554 \\
(0,085) \\
\end{array}$ & $\dagger$ \\
\hline Facilidades de apertura & $\begin{array}{l}-0,004 \\
(0,004)\end{array}$ & & $\begin{array}{c}-0,019 \\
(0,009) \\
\end{array}$ & * & $\begin{array}{c}0,001 \\
(0,007) \\
\end{array}$ & & $\begin{array}{l}-0,004 \\
(0,006) \\
\end{array}$ & \\
\hline Cortes de energía & $\begin{array}{c}0,546 \\
(0,290) \\
\end{array}$ & & $\begin{array}{c}0,140 \\
(0,163) \\
\end{array}$ & & $\begin{array}{l}-0,198 \\
(0,204) \\
\end{array}$ & & $\begin{array}{c}0,129 \\
(0,067) \\
\end{array}$ & * \\
\hline Uso de recursos propios & $\begin{array}{c}0,091 \\
(0,471)\end{array}$ & & $\begin{array}{c}0,292 \\
(0,319) \\
\end{array}$ & & $\begin{array}{l}-0,047 \\
(0,162) \\
\end{array}$ & & $\begin{array}{c}0,122 \\
(0,069) \\
\end{array}$ & \\
\hline Uso de recursos bancarios & $\begin{array}{c}-0,572 \\
(0,213) \\
\end{array}$ & $\ddagger$ & $\begin{array}{c}0,073 \\
(0,236) \\
\end{array}$ & & $\begin{array}{l}-0,106 \\
(0,074) \\
\end{array}$ & & $\begin{array}{c}0,207 \\
(0,121) \\
\end{array}$ & \\
\hline Uso de crédito comercial & $\begin{array}{c}0,370 \\
(0,168) \\
\end{array}$ & * & $\begin{array}{c}-0,231 \\
(0,325) \\
\end{array}$ & & $\begin{array}{c}0,296 \\
(0,107) \\
\end{array}$ & $\ddagger$ & $\begin{array}{c}0,254 \\
(0,203) \\
\end{array}$ & \\
\hline Uso de otros recursos & $\begin{array}{c}0,081 \\
(0,225) \\
\end{array}$ & & $\begin{array}{c}0,450 \\
(0,452) \\
\end{array}$ & & $\begin{array}{l}-0,186 \\
(0,144) \\
\end{array}$ & & $\begin{array}{c}-0,060 \\
(0,104) \\
\end{array}$ & \\
\hline Constante & $\begin{array}{c}1,669 \\
(0,431)\end{array}$ & $\dagger$ & $\begin{array}{c}0,500 \\
(0,944) \\
\end{array}$ & & $\begin{array}{c}2,519 \\
(1,368) \\
\end{array}$ & * & $\begin{array}{l}-1,039 \\
(0,218) \\
\end{array}$ & $\dagger$ \\
\hline Observaciones & 161 & & 158 & & 1,213 & & 1,988 & \\
\hline R2 (ajustada) & 0,682 & & 0,707 & & 0,736 & & 0,760 & \\
\hline
\end{tabular}

Notas: Estimaciones utilizando el método de mínimos cuadrados en dos etapas. Las variables instrumentadas fueron las referentes a los activos fijos (cap) y los trabajadores permanentes (tra). En todas las especificaciones se incluyen como variables independientes el crecimiento del PIB y la inflación, se incluyen también dummies por industria. Se reportan entre paréntesis los errores estándar, estimados agrupando -cluster- a las empresas por la industria a la que pertenecen. Coeficientes estadísticamente significativos al: $1 \% t$, $5 \% \neq$ y $10 \%$ *

Fuente: Estimaciones propias del autor con base en datos de la Enterprise Survey del Banco Mundial, Estadísticas del Banco Mundial y Estadísticas Monetarias y Financieras del Fondo Monetario Internacional. 
Los resultados del Cuadro 4 sugieren una relativa diferencia en el comportamiento de las empresas según el grado de competencia en el mercado. Así, aún y cuando en la mayoría de estas especificaciones el coeficiente de la variable sobre acceso al sistema financiero es positivo, sólo para la sub-muestra de empresas que respondió tener más de cinco competidores es significativo estadísticamente, por lo tanto no es evidente la relación positiva que se esperaba tuviese esta variable con el desempeño de las empresas.

En lo referente a las relaciones entre las diferentes fuentes de recursos y el desempeño de las empresas, los resultados de del Cuadro 4 son poco evidentes. Ello debido a que aún y cuando hay diferencias en los signos de los coeficientes de acuerdo al nivel de competencia en el mercado, en la mayoría de los casos éstos resultaron no ser estadísticamente significativos. En este sentido, con los resultados este cuadro, no es posible establecer un patrón de comportamiento entre las empresas que enfrentan diferentes grados de competencia. Lo cual podría ser consecuencia de la propia construcción de los proxies de la competencia en el mercado, pues como Boone (2008) menciona, la menor concentración, y por lo tanto un mayor número de empresas en el mercado, no siempre captura la existencia de presiones competitivas.

Por su parte, los resultados del Cuadro 5, a diferencia de los resultados mostrados en el Cuadro 4, sí sugieren un comportamiento diferenciado entre las empresas que enfrentan a mayores o menores presiones competitivas. Así, para las empresas que introdujeron nuevos productos, el coeficiente del acceso al sistema financiero es mayor, y altamente significativo estadísticamente, esto contrasta con el coeficiente hallado en el caso de las empresas que no introdujeron o mejoraron sus productos. Algo similar sucede al considerar la diferencia entre las empresas que realizaron mejoras en los procesos y aquéllas que no.

De esta manera, los resultados del Cuadro 5 sugieren que el acceso al sistema financiero favorece más el desempeño de las empresas que perciben mayores presiones competitivas en el mercado. Con lo cual, los resultados hallados podrían sugerir una relación positiva entre el acceso al sistema financiero y un mejor desempeño de las empresas. Ello puede ser resultado de la relativa disponibilidad de recursos para ampliar la producción de las empresas, y así, bien permanecer en el mercado al mejorar los productos y procesos que les permitan enfrentar la competencia, o en su caso con las mejoras en productos y procesos provocar la posible salida de competidores en el mercado.

Por otra parte, ante la percepción de mayores presiones competitivas, los resultados sugieren que las empresas prefieren demandar más crédito comercial para financiar su capital de trabajo. Lo anterior, se ve reflejado al comparar los niveles de los coeficientes del crédito comercial, los cuales en el caso de empresas que perciben mayores presiones competitivas superan en alrededor de tres veces los niveles de los coeficientes de empresas con menores presiones competitivas. 


\section{Cuadro 5}

Desempeño, decisiones financieras e innovación y competencia ante diferente grado de competencia

\begin{tabular}{|c|c|c|c|c|c|c|c|c|}
\hline \multirow[b]{2}{*}{ Activos fijos } & \multicolumn{2}{|c|}{$\begin{array}{c}\text { Mejoraron o } \\
\text { introdujeron } \\
\text { nuevos } \\
\text { productos }\end{array}$} & \multicolumn{2}{|c|}{$\begin{array}{c}\text { No mejoraron } \\
\text { o introdujeron } \\
\text { nuevos } \\
\text { productos }\end{array}$} & \multicolumn{2}{|c|}{$\begin{array}{l}\text { Mejoraron } \\
\text { procesos }\end{array}$} & \multicolumn{2}{|c|}{$\begin{array}{c}\text { No mejoraron } \\
\text { procesos }\end{array}$} \\
\hline & $\begin{array}{r}0,375 \\
(0,102) \\
\end{array}$ & $\dagger$ & $\begin{array}{r}0,393 \\
(0,133) \\
\end{array}$ & $\ddagger$ & $\begin{array}{r}0,527 \\
(0,263) \\
\end{array}$ & * & $\begin{array}{r}0,290 \\
(0,137) \\
\end{array}$ & * \\
\hline Trabajadores permanentes & $\begin{array}{r}0,629 \\
(0,193)\end{array}$ & $\ddagger$ & $\begin{array}{r}0,748 \\
(0,159)\end{array}$ & $\dagger$ & $\begin{array}{r}0,406 \\
(0,318)\end{array}$ & & $\begin{array}{r}0,949 \\
(0,159)\end{array}$ & $\dagger$ \\
\hline Actividad exportadora & $\begin{array}{r}0,327 \\
(0,161) \\
\end{array}$ & * & $\begin{array}{r}0,298 \\
(0,176) \\
\end{array}$ & & $\begin{array}{r}0,422 \\
(0,165) \\
\end{array}$ & $\ddagger$ & $\begin{array}{r}0,136 \\
(0,112) \\
\end{array}$ & \\
\hline Experiencia administrador & $\begin{array}{r}-0,011 \\
(0,005) \\
\end{array}$ & * & $\begin{array}{r}-0,003 \\
(0,004) \\
\end{array}$ & & $\begin{array}{r}-0,017 \\
(0,006) \\
\end{array}$ & $\ddagger$ & $\begin{array}{r}0,003 \\
(0,005) \\
\end{array}$ & \\
\hline Acceso al sistema financiero & $\begin{array}{r}0,579 \\
(0,123) \\
\end{array}$ & $\dagger$ & $\begin{array}{r}0,323 \\
(0,213) \\
\end{array}$ & & $\begin{array}{r}0,623 \\
(0,138) \\
\end{array}$ & $\dagger$ & $\begin{array}{r}0,346 \\
(0,157) \\
\end{array}$ & * \\
\hline Facilidades de apertura & $\begin{array}{r}-0,006 \\
(0,005) \\
\end{array}$ & & $\begin{array}{r}0,001 \\
(0,003) \\
\end{array}$ & & $\begin{array}{r}-0,009 \\
(0,003) \\
\end{array}$ & $\ddagger$ & $\begin{array}{r}-0,002 \\
(0,006) \\
\end{array}$ & \\
\hline Cortes de energía & $\begin{array}{r}-0,029 \\
(0,118) \\
\end{array}$ & & $\begin{array}{r}0,095 \\
(0,041) \\
\end{array}$ & $\ddagger$ & $\begin{array}{r}-0,005 \\
(0,072) \\
\end{array}$ & & $\begin{array}{r}0,023 \\
(0,101) \\
\end{array}$ & \\
\hline Uso de recursos propios & $\begin{array}{r}-0,002 \\
(0,046) \\
\end{array}$ & & $\begin{array}{r}0,034 \\
(0,076) \\
\end{array}$ & & $\begin{array}{r}0,046 \\
(0,097) \\
\end{array}$ & & $\begin{array}{r}-0,098 \\
(0,058) \\
\end{array}$ & \\
\hline Uso de recursos bancarios & $\begin{array}{r}0,099 \\
(0,072) \\
\end{array}$ & & $\begin{array}{r}-0,134 \\
(0,146) \\
\end{array}$ & & $\begin{array}{r}0,005 \\
(0,102) \\
\end{array}$ & & $\begin{array}{r}0,084 \\
(0,142) \\
\end{array}$ & \\
\hline Uso de crédito comercial & $\begin{array}{r}0,422 \\
(0,105) \\
\end{array}$ & $\dagger$ & $\begin{array}{r}0,138 \\
(0,057) \\
\end{array}$ & $\ddagger$ & $\begin{array}{r}0,444 \\
(0,155) \\
\end{array}$ & $\ddagger$ & $\begin{array}{r}0,184 \\
(0,080) \\
\end{array}$ & $\ddagger$ \\
\hline Uso de otros recursos & $\begin{array}{r}-0,197 \\
(0,065) \\
\end{array}$ & $\ddagger$ & $\begin{array}{r}0,189 \\
(0,103) \\
\end{array}$ & & $\begin{array}{r}-0,049 \\
(0,130) \\
\end{array}$ & & $\begin{array}{r}-0,029 \\
(0,130) \\
\end{array}$ & \\
\hline Constante & $\begin{array}{r}2,357 \\
(0,773) \\
\end{array}$ & $\ddagger$ & $\begin{array}{r}0,904 \\
(0,388) \\
\end{array}$ & $\ddagger$ & $\begin{array}{r}2,899 \\
(1,017) \\
\end{array}$ & $\ddagger$ & $\begin{array}{r}-0,886 \\
(0,295) \\
\end{array}$ & $\ddagger$ \\
\hline Observaciones & 2.544 & & 1.382 & & 2.460 & & 1.466 & \\
\hline R2 (ajustada) & 0,759 & & 0,671 & & 0,737 & & 0,678 & \\
\hline
\end{tabular}

Notas: Estimaciones utilizando el método de mínimos cuadrados en dos etapas. Las variables instrumentadas fueron las referentes a los activos fijos (cap) y los trabajadores permanentes (tra). En todas las especificaciones se incluyen como variables independientes el crecimiento del PIB y la inflación, se incluyen también dummies por industria. Se reportan entre paréntesis los errores estándar, estimados agrupando -cluster- a las empresas por la industria a la que pertenecen. Coeficientes estadísticamente significativos al: $1 \% t$, $5 \% \neq$ y $10 \%$ *.

Fuente: Estimaciones propias del autor con base en datos de la Enterprise Survey del Banco Mundial, Estadísticas del Banco Mundial y Estadísticas Monetarias y Financieras del Fondo Monetario Internacional.

El mayor uso relativo del crédito comercial ante la percepción de presiones competitivas, podría ser explicado por las características propias de este instrumento, puesto que éste se considera una fuente informal de recursos y depende de las relaciones de negocios que las empresas establecen con sus proveedores y clientes. ${ }^{16}$ Ante ello, la obtención de crédito de parte de los proveedores puede

${ }^{16}$ Sobre los determinantes del crédito comercial existe literatura relativamente extensa. Petersen y Rajan (1997) documentan los principales motivos que originan la oferta y demanda del crédito comercial. 
ser relativamente más sencilla, en comparación con la obtención de recursos provenientes de otras fuentes, como por ejemplo los recursos de la banca. ${ }^{17}$

Los resultados hallados en las diversas estimaciones muestran que las relaciones de la competencia enfrentada en el mercado con el desempeño de las empresas no son del todo evidentes. Es decir, bajo algunas circunstancias la mayor competencia guarda una relación positiva, mientras que bajo otras condiciones la competencia pareciera que disminuye los beneficios de las empresas. Este hecho coincide con lo propuesto, y explicado, por Aghion y Griffith (2005), quienes muestran la existencia de efectos contrarios de la competencia en el mercado sobre el crecimiento de las empresas.

Por otra parte, los resultados sugieren que la interacción entre las presiones competitivas en el mercado percibidas por las empresas y sus decisiones financieras, influyen relativamente más sobre el desempeño de las empresas que interactúan en entornos competitivos "más agresivos". Asimismo, este hecho podría estar sugiriendo un uso relativamente mayor de los diversos instrumentos financieros como un instrumento de competencia por parte estas empresas. Por lo que este hecho se asocia con lo propuesto por la reciente literatura que vincula las decisiones financieras y la competencia enfrentada en el mercado, la cual sugiere que las decisiones financieras pueden ser consideradas como parte de los instrumentos incluidos en las estrategias competitivas de las empresas.

\section{CONCLUSIONES}

El desempeño de las empresas resulta de las decisiones que adoptan en los diferentes mercados donde interactúan. Con el fin de analizar las relaciones entre las decisiones financieras y la competencia en el mercado, a diferencia de trabajos previos, en este documento se exploran de manera conjunta las relaciones que ambos tipos de decisiones guardan con el desempeño de las empresas, para ello se utilizan los datos de la Enterprise Survey, del Banco Mundial, para poco más de 3.500 empresas distribuidas en 14 países iberoamericanos.

Los resultados de este trabajo, sugieren que al aproximar las presiones competitivas a través del número de competidores en el mercado, ésta guarda una relación en forma de "U invertida" respecto al desempeño de las empresas. Es decir, que la competencia "moderada" en el mercado podría tener un mayor efecto que la ausencia de competencia o la competencia en "exceso". Por otra parte, al medir las presiones competitivas a través de proxies relacionados con el proceso de innovación es posible apreciar una relación negativa, aunque poco significativa estadísticamente.

\footnotetext{
${ }^{17}$ Fisman (2001) propone que en economías con sistemas financieros "débiles" el crédito comercial favorece la eficiencia productiva de las empresas.
} 
Respecto a las decisiones financieras de las empresas y sus efectos sobre el desempeño de las empresas, los resultados presentados sugieren una relación positiva entre el acceso al sistema financiero y el desempeño de las empresas. Estos resultados son consistentes con los diversos resultados obtenidos en diferentes trabajos previos sobre el impacto del desarrollo financiero en el crecimiento. Por otra parte, los resultados hallados muestran la relativa importancia del crédito comercial como una de las principales fuentes de financiamiento de las empresas, este hecho es consistente con lo sugerido en la literatura previa.

Los resultados de los ejercicios econométricos que pretenden aislar las decisiones financieras de las empresas bajo entornos con diferentes presiones competitivas muestran que generalmente para las empresas que perciben mayores presiones competitivas en el mercado, los posibles efectos de sus decisiones financieras son superiores a las de aquellas empresas con una percepción menor de competencia en el mercado. De esta manera, los resultados sugieren que las decisiones financieras podrían estar siendo utilizadas como un instrumento adicional para competir en el mercado. Dentro de las decisiones financieras, destaca el uso relativamente mayor del crédito comercial entre las empresas que perciben mayor competencia en el mercado.

Como cualquier otro trabajo de investigación este documento tiene algunas limitantes, dentro de las limitantes pueden mencionarse la falta de datos de las empresas en más periodos, es decir contar con una estructura de datos de panel, lo cual impide incorporar el carácter dinámico al análisis; la propia naturaleza de los datos utilizados -encuestas- que puede incidir sobre la existencia de un sesgo subjetivo y; no obstante lo argumentado en la selección de los proxies de la competencia en el mercado, éstos pueden ser sujetos de crítica y mejora.

Este documento también aporta una agenda de investigación, quedando pendientes para futuros trabajos algunos tópicos no abordados. Una primera extensión de este documento es el análisis de los efectos de las decisiones financieras y la competencia en el mercado considerando el tamaño de las empresas. Un trabajo futuro adicional, podría profundizar sobre las diferencias en el comportamiento de las empresas de acuerdo a la industria donde interactúan. Por último, cabe mencionar que este documento, no obstante las posibles limitaciones señaladas, ayuda a cubrir la falta de evidencia empírica para el caso de empresas en Iberoamérica sobre la interrelación existente entre las decisiones financieras de las empresas y el entorno competitivo enfrentado. 


\section{REFERENCIAS BIBLIOGRÁFICAS}

AGHION, P. y GRIFFITH, R. (2005). Competition and Growth. Reconciling Theory and Evidence, MIT Press.

AGHION, P.; BLOOM, N.; BLUNDELL, R.; GRIFFITH, R. y HOWITT, P. (2005). "Competition and innovation: An inverted-u relationship". Quarterly Journal of Economics, 120(2), pp. 701-728.

AGHION, P.; BRAUN, M; y FEDDERKE, J. (2008). "Competition and productivity growth in South Africa". Economics of Transition, 16(4), pp. 741768.

AYBAR-ARIAS, C.; CASINO-MARTINEZ, A; y LÓPEZ-GRACIA, J. (2003). "Estrategia y estructura de capital en la PYME: Una aproximación empírica". Estudios de Economía Aplicada, 21(1), pp. 27-52.

BANCO MUNDIAL (2007) "Enterprise survey. Understanding the sampling methodology", Documentos metodológicos de la Encuesta de Empresas, 15 de enero de 2007, disponible en www.enterprisesurveys.org.

BECK, T.; DEMIRGUÇ-KUNT, A. y MAKSIMOVIC, V. (2008). "Financing patterns around the world: Are small firms different?". Journal of Financial Economics, 89(3), pp. 467-487.

BOONE, J. (2008). "Competition: Theoretical parameterizations and empirical measures". Journal of Institutional and Theoretical Economics, 164(4), pp. 587-611.

BROWN, G., CHAVIS L. y KLAPPER L. (2008). "A new lease on life: Institutions, external finance and business growth", AFA 2008 New Orleans, Meeting Papers.

BUTTLER, A. y CORNAGGIA, J. (2008). "Does access to external finance improve productivity? Evidence from a natural experiment", Dallas School of Management, University of Texas.

CARLIN, W.; FRIES, S.; SCHAFFER, M. y SEABRIGHT, P. (2001). "Competition and enterprise performance in transition economies. Evidence from a cross country survey", CEPR, Discussion paper Núm. 2840.

CARLIN, W.; SCHAFFER, M. y SEABRIGHT, P. (2004). "A minimum of rivalry: Evidence from transition economies on the importance of competition for innovation and growth", The William Davidson Institute, University of Michigan, Working Paper Núm. 670.

COAD, A. y HÖLZL, W. (2010) "Firm growth: empirical analysis", Max Planck Institute of Economics, Papers on Economics and Evolution Núm. 1002.

COMMANDER, S. y SVEJNAR, J. (2008). "Do institutions, ownership, exporting and competition explain firm performance?", International Policy Center, Gerald R. Ford School of Public Policy, University of Michigan, Working Paper Series Núm. 65. 
CULL, R.; XU, L.C. y ZHU, T. (2009). "Formal finance and trade credit during China's transition". Journal of Financial Intermediation, 18(2), pp. 173-192.

DEMIRGUC-KUNT, A. y MAKSIMOVIC, V. (2001). "Firms as financial intermediaries: Evidence from trade credit data", Banco Mundial, Policy Research Working Paper Núm. 2696.

FISMAN, R. (2001). "Trade credit and productive efficiency in developing countries". World Development, 29(2), pp. 311-321.

FISMAN, R. y LOVE, I. (2003). "Trade credit, financial intermediary development, and industry growth". Journal of Finance, 58(1), pp. 353-374.

GATTI, R. y LOVE, I. (2008). "Does access to credit improve productivity?. Evidence from Bulgarian firms". Economics of Transition, 16(3), pp. 445-465.

$\mathrm{GOHSH}, \mathrm{S}$. (2009). "Productivity and financial structure: Evidence from Indian high-tech firms", MPRA paper Núm. 19467.

JONG, A., NGUYEN, T. T. y VAN DICK, M. (2007). "Strategic debt: Evidence from Bertrand and Cournot competition", Report Series Research in Management ERS-2007-057-F\&A, Erasmus Research Institute of Management, Erasmus University.

LEVINE, R. (2004). "Finance and growth: Theory and evidence", NBER, Working Paper Núm. 10766.

LI, D. y LU, Y. (2008). "Does trade credit really help? Evidence from China", Documento presentado en Far Eastern and South Asian Meting of Econometrics 2008, Singapore Management University.

LYANDRES, E. (2006). "Capital structure and interaction among firms in output markets: Theory and evidence", Journal of Business, 79(5), 2381-2421.

MAROTO, J., MELLE, M., MORENO, I. y RODRÍGUEZ, J. (2006). "Grado de competencia, presión de la deuda y productividad empresarial: Un análisis empírico desde la perspectiva del gobierno corporativo", Panorama Socioeconómico, 24(33), 8-17.

MURRAY, M.P. (2006). "Avoiding invalid instruments and coping with weak instruments". Journal of Economic Perspectives, 20(4), 111-132.

NEFF, C. (2003). Corporate Finance, Innovation, and Strategic Competition, Lecture Notes in Economics and Mathematical Systems, Núm. 522, Springer.

NICKELL, S. (1996). "Competition and corporate performance". Journal of Political Economy, 104(4), pp. 724-746.

NICKELL, S.; NICOLITSAS, D. y DRYDEN, N. (1997) "What makes firms perform well?", European Economic Review, 43(3), 783-796.

OSPINA, S. y SCHIFFBAUER, M. (2010). "Competition and firm productivity: Evidence from firm-level data", Fondo Monetario Internacional, Working Paper 10/67.

PETERSEN, M. y RAJAN, R. (1997). "Trade credit: Theories and evidence", The Review of Financial Studies, 10(3), 661-691. 
SAEED, A. (2009). "Does nature of financial institutions matter to firm growth in transition economies?". Eurasian Journal of Business and Economics, 2(3), pp. 73-90.

SALAS-FUMÁS, V. (1996). "Economía y gestión de los activos intangibles", Economía industrial, 307, 17-24.

SALGADO-BANDA, H. y BERNAL-VERDUGO, L. (2007). "Multifactor productivity and its determinants: An empirical analysis for Mexican manufacturing", Banco de México, Documento de Investigación Núm. 2007-09.

SÁNCHEZ-VALADEZ, M. (2009). "Endeudamiento y competencia en el mercado: Evidencia de las empresas en México", Revista Economía: Teoría y Práctica, 31, 9-42.

SÁNCHEZ-VALADEZ, M. (2010). "Trade credit and market competition. Some facts for firms in the Mexican economy", Documento presentado en la 6th International Student Conference: Empirical Studies in Social Sciences, Izmir University of Economics, Turquía.

SHOWALTER, D. (1999). "Strategic debt: evidence in manufacturing", International Journal of Industrial Organization, 17(3), 319-333.

TANG, J. y WANG, W. (2002). "Competition and productivity: Evidence from Canadian manufacturing firms", Micro Economic and Policy Analysis Branch. Industry Canada. 


\begin{tabular}{|c|c|c|}
\hline \multicolumn{3}{|c|}{$\begin{array}{c}\text { Cuadro } 6 \\
\text { Variables utilizadas en las estimaciones }\end{array}$} \\
\hline Variables & Definición & Fuente \\
\hline \multicolumn{3}{|l|}{ Variable dependiente } \\
\hline vtas & Logaritmo de las ventas expresadas en dólares de Estados Unidos. & BMEE \\
\hline \multicolumn{3}{|c|}{ Variables independientes } \\
\hline \multicolumn{3}{|c|}{ Insumos principales } \\
\hline cap & $\begin{array}{l}\text { Logaritmo del valor de los activos fijos expresados en dólares de } \\
\text { Estados Unidos. }\end{array}$ & BMEE \\
\hline trab & Logaritmo del total de trabajadores permanentes. & BMEE \\
\hline \multicolumn{3}{|c|}{$\begin{array}{l}\text { Características de la } \\
\text { empresa }\end{array}$} \\
\hline $\exp$ & Dummy igual a 1 si la empresa tiene exportaciones directas o indirectas. & BMEE \\
\hline admn & Años de experiencia del administrador. & BMEE \\
\hline \multicolumn{3}{|l|}{ Acceso a recursos } \\
\hline accfin & Dummy igual a 1 si la empresa tiene la posibilidad de sobregiro. & BMEE \\
\hline apert & Días necesarios para abrir un negocio & $\mathrm{BM}$ \\
\hline ener & $\begin{array}{l}\text { Dummy igual a } 1 \text { si la empresa tuvo cortes del suministro de electricidad } \\
\text { o agua. }\end{array}$ & BMEE \\
\hline \multicolumn{3}{|l|}{ Variables macro } \\
\hline cpnb & Tasa de crecimiento anual del PIB en 2006. & BM \\
\hline inf & Inflación en 2006. & FMI \\
\hline \multicolumn{3}{|c|}{ Decisiones financieras } \\
\hline ct_propios & $\begin{array}{l}\text { Dummy igual a } 1 \text { si la empresa utilizó recursos propios para financiar su } \\
\text { capital de trabajo. }\end{array}$ & BMEE \\
\hline ct_bcos & $\begin{array}{l}\text { Dummy igual a } 1 \text { si la empresa utilizó recursos de bancos privados o } \\
\text { estatales para financiar su capital de trabajo. }\end{array}$ & BMEE \\
\hline ct_crcom & $\begin{array}{l}\text { Dummy igual a } 1 \text { si la empresa utilizó crédito comercial para financiar su } \\
\text { capital de trabajo. }\end{array}$ & BMEE \\
\hline ct_otras & $\begin{array}{l}\text { Dummy igual a } 1 \text { si la empresa utilizó recursos de otras fuentes } \\
\text { informales para financiar su capital de trabajo. }\end{array}$ & BMEE \\
\hline \multicolumn{3}{|l|}{ Competencia } \\
\hline comp & Número de competidores en el mercado. & BMEE \\
\hline comp1 & No enfrentan competidores en el mercado. & BMEE \\
\hline comp2 & Enfrenta 1 competidor en el mercado. & BMEE \\
\hline comp3 & Enfrenta entre 2 y 5 competidores en el mercado. & BMEE \\
\hline comp4 & Enfrenta más de 5 competidores en el mercado. & BMEE \\
\hline nprod & Dummy igual a 1 si hubo mejora o introducción de nuevos productos. & BMEE \\
\hline nproc & Dummy igual a 1 si hubo mejora de procesos. & BMEE \\
\hline \multicolumn{3}{|c|}{$\begin{array}{l}\text { Instrumentos de cap y } \\
\text { trab }\end{array}$} \\
\hline edad1 & Dummy igual a 1 si la empresa tiene menos de 6 años en el mercado. & BMEE \\
\hline edad2 & Dummy igual a 1 si la empresa tiene entre 6 y 15 años en el mercado. & BMEE \\
\hline edad3 & Dummy igual a 1 si la empresa tiene más de 15 años en el mercado. & BMEE \\
\hline edu1 & Porcentaje de trabajadores cualificados en la empresa. & BMEE \\
\hline dciud & Dummy igual a 1 si la empresa se localiza en ciudad capital del país. & BMEE \\
\hline trabt3 & Logaritmo del número de trabajadores permanentes 3 años antes. & BMEE \\
\hline
\end{tabular}

Fuente: BMEE: Banco Mundial, Encuesta de las Empresas - Enterprise Survey; BM: Estadísticas del Banco Mundial; FMl: Fondo Monetario Internacional, Estadísticas Monetarias y Financieras. 


\section{Cuadro 7}

Estadísticas de las variables utilizadas

\begin{tabular}{|c|c|c|c|c|c|c|c|}
\hline & Obs. & Media & Desv. Est. & & Obs. & Media & $\begin{array}{l}\text { Desv. } \\
\text { Est. }\end{array}$ \\
\hline Variables dependientes & & & & $\begin{array}{l}\text { Decisiones } \\
\text { financieras }\end{array}$ & & & \\
\hline vtas & 3.512 & 6,13 & 2,01 & ct_propios & 3.512 & 0,85 & 0,36 \\
\hline Insumos principales & & & & ct_bcos & 3.512 & 0,39 & 0,49 \\
\hline cap & 3.512 & 4,84 & 2,12 & ct_crcom & 3.512 & 0,39 & 0,49 \\
\hline trab & 3.512 & 3,25 & 1,25 & ct_otras & 3.512 & 0,20 & 0,40 \\
\hline Características de la empresa & & & & Competencia & & & \\
\hline $\exp$ & 3.512 & 0,27 & 0,44 & comp & 3.512 & 3,90 & 1,42 \\
\hline admn & 3.512 & 21,72 & 11,83 & comp1 & 3.512 & 0,05 & 0,21 \\
\hline edad & 3.512 & 24,88 & 19,13 & comp2 & 3.512 & 0,04 & 0,21 \\
\hline edu1 & 3.512 & 43,83 & 28,53 & comp3 & 3.512 & 0,34 & 0,48 \\
\hline trabt3 & 3.512 & 68,87 & 315,59 & comp4 & 3.512 & 0,57 & 0,50 \\
\hline Acceso a recursos & & & & nprod & 3.512 & 0,64 & 0,48 \\
\hline accfin & 3.512 & 0,60 & 0,49 & nproc & 3.512 & 0,61 & 0,49 \\
\hline ener & 3.512 & 0,55 & 0,50 & & & & \\
\hline
\end{tabular}

Fuente: Elaboración propia a partir de los datos de la Enterprise Survey del Banco Mundial. 
